\title{
Large-domain stability of random attractors for stochastic $g$-Navier-Stokes equations with additive noise
}

\author{
Fuzhi Li ${ }^{1}$, Dongmei $\mathrm{Xu}^{1 *}$ (D) and Lianbing She ${ }^{2}$
}

\section{"Correspondence:}

xudongmei@126.com

'School of Mathematics and

Computer Science, Shangrao

Normal University, Shangrao

334001, P.R. China

Full list of author information is

available at the end of the article

\section{Springer}

\begin{abstract}
This paper concerns the long term behavior of the stochastic two-dimensional $g$-Navier-Stokes equations with additive noise defined on a sequence of expanding domains, where the ultimate domain is unbounded and of Poincaré type. We prove that the weak continuity is uniform with respect to all expanding cocycles, which yields the equi-asymptotic compactness by using an energy equation method. Finally, we show the existence of a random attractor for the equation on each domain and the upper semi-continuity of random attractors as the bounded domain is expanded to the unbounded ultimate domain.
\end{abstract}

MSC: 35B40; 35B41; 37L30

Keywords: Stochastic g-Navier-Stokes; Expanding domains; Expanding cocycles; Energy equation method; Equi-asymptotic compactness; Upper semi-continuity

\section{Introduction}

The fluid dynamics of deterministic or stochastic Navier-Stokes (NS) equations has been extensively studied. For example, many properties such as existence, upper semicontinuity, regularity, and fractal dimension of an attractor were studied in the literature $[4,5,8,13,19]$. However, we find that most of the above-mentioned studies are given in a two-dimensional situation rather than three-dimensional one, which encourages us to do more in-depth research about the dynamic behavior of Navier-Stokes equations.

The $g$-NS equations in spatial dimension 2 were introduced by Roh [18] as follows:

$$
\left\{\begin{array}{l}
\frac{\partial u}{\partial t}-v \Delta u+(u \cdot \nabla) u+\nabla p=f(x), \\
\nabla \cdot(g u)=0
\end{array}\right.
$$

where $g$ is a suitable smooth real-valued function. The uniqueness and existence of solutions for the $g$-NS equations in $\mathbb{R}^{n}(n=2,3)$ were proved by Bae and Roh [3]. When $g=1$, Eq. (1) becomes the usual 2D Navier-Stokes equations. In the last decade, the limiting behavior of solutions in terms of the existence of attractors for 2D $g$-NS equations has been

(c) The Author(s) 2020. This article is licensed under a Creative Commons Attribution 4.0 International License, which permits use sharing, adaptation, distribution and reproduction in any medium or format, as long as you give appropriate credit to the original author(s) and the source, provide a link to the Creative Commons licence, and indicate if changes were made. The images or other third party material in this article are included in the article's Creative Commons licence, unless indicated otherwise in a credit line to the material. If material is not included in the article's Creative Commons licence and your intended use is not permitted by statutory regulation or exceeds the permitted use, you will need to obtain permission directly from the copyright holder. To view a copy of this licence, visit http://creativecommons.org/licenses/by/4.0/. 
studied in both autonomous and non-autonomous cases without the stochastic situations, see $[10,11,18]$.

As described in [18], the 2D $g$-NS equations arise in a natural way when we study the standard 3D Navier-Stokes problem in a 3D thin domain $\mathcal{O}_{g}=\mathcal{O} \times(0, \varepsilon g),\left(\mathcal{O} \subset \mathbb{R}^{2}\right)$ which was introduced by $[9,17]$, and we do not claim that the $g$-NS equations form a model of any fluid flow. They may, or may not. That they are derived from a standard 3D problem is the basis for our study. However, as we know, there are no results related to the long-time behavior of solutions for the 2D stochastic $g$-NS equations.

In this paper, we consider both the existence and large-domain stability of a random attractor for the stochastic 2D $g$-NS equations on an unbounded Poincaré-type domain $\mathcal{O}_{\infty} \subset \mathbb{R}^{2}$, which is regarded as a limit of the sequence of expanding domains $\mathcal{O}_{k}=\{x \in$ $\left.\mathcal{O}_{\infty}:|x|<k\right\}$.

We write the sequence of stochastic $2 \mathrm{D} g$-NS equations on $\mathcal{O}_{k}(k \in \overline{\mathbb{N}}:=\mathbb{N} \cup\{\infty\})$ as a unified form:

$$
\left\{\begin{array}{l}
d u_{k}-\left(v \Delta u_{k}-\left(u_{k} \cdot \nabla\right) u_{k}-\nabla p\right) d t=f(x, t) d t+\varepsilon h(x) d W(t), \quad x \in \mathcal{O}_{k}, \\
\nabla \cdot\left(g u_{k}\right)=0, \quad x \in \mathcal{O}_{k}, \\
u_{k}=0, \quad x \in \partial \mathcal{O}_{k}, \\
u_{k}(\tau, x)=u_{\tau, k}(x), \quad x \in \mathcal{O}_{k}, \tau \in \mathbb{R},
\end{array}\right.
$$

where $v, \varepsilon>0, p$ is the pressure, $u_{k}$ is the velocity vector, $W$ is a scalar Wiener process defined on a probability space $(\Omega, \mathcal{F}, P) . h(x)$ is a given time-independent two-dimensional vector function belonging to some Sobolev spaces which will be specified later.

The first subject is to show the existence of a random attractor $\mathcal{A}_{k}$ in $H_{g}\left(\mathcal{O}_{k}\right)$ (a special subspace of $\mathbb{L}^{2}\left(\mathcal{O}_{k}\right)$ ) for each $k \in \overline{\mathbb{N}}$. Due to both non-autonomy and randomness of model (2), the attractor is actually a bi-parametric set $\mathcal{A}_{k}=\left\{\mathcal{A}_{k}(\tau, \omega): \tau \in \mathbb{R}, \omega \in \Omega\right\}$ in $H_{g}\left(\mathcal{O}_{k}\right)$ (see [21]). Even for this existence of a pullback attractor, the assumption of small noise $\left(\varepsilon \leq \varepsilon_{0}\right)$ seems to be necessary.

To study problem (2), the real-valued function $g=g(x) \in W^{1, \infty}\left(\mathcal{O}_{\infty}\right)$ satisfies the following basic assumption:

$$
0<m_{0} \leq g(x) \leq M_{0}, \quad \forall x=\left(x_{1}, x_{2}\right) \in \mathcal{O}_{\infty}
$$

Using the famous energy equation method [19], we establish the existence of random attractors. More precisely, for each $k \in \overline{\mathbb{N}}$, the stochastic $g$-NS equations (2) have a random attractor $\mathcal{A}_{k}$ in $H_{g}\left(\mathcal{O}_{k}\right)$.

The second subject is to investigate large-domain stability of the attractor, which means that $\mathcal{A}_{k}$ is stable (upper semi-continuous) at $\mathcal{A}_{\infty}$ under a suitable Hausdorff semi-distance.

Such an expanding-domain problem is contrary to the thin-domain problem, the latter was extensively investigated in the literature (see $[14,15])$ and time-varying domains problem [20]. However, the same difficulty arises from the fact that both $\mathcal{A}_{k}$ and $\mathcal{A}_{\infty}$ lie in different phase spaces, compared with the same phase space in time-dependent stability of a pullback attractor $[6,7,12]$.

In order to define a distance between two subsets lying in different spaces, we consider the null-expansion $\widetilde{u_{k}}$ of the solution $u_{k} \in H_{g}\left(\mathcal{O}_{k}\right)$ defined by

$$
\tilde{u_{k}}=u_{k}, \quad x \in \mathcal{O}_{k} ; \quad \tilde{u_{k}}=0, \quad x \in \mathcal{O}_{\infty} \backslash \mathcal{O}_{k} .
$$


One can show easily that $\tilde{u_{k}} \in H_{g}\left(\mathcal{O}_{\infty}\right)$ if $u_{k} \in H_{g}\left(\mathcal{O}_{k}\right)$. However, in general, $\tilde{u_{k}} \neq u_{\infty}$. So, the attractor $\mathcal{A}_{k}$ can be expanded to a bi-parametric set $\widetilde{\mathcal{A}_{k}}$ in $H_{g}\left(\mathcal{O}_{\infty}\right)$, defined by

$$
\widetilde{\mathcal{A}_{k}}(\tau, \omega)=\left\{u \in H_{g}\left(\mathcal{O}_{\infty}\right): \exists v \in \mathcal{A}_{k}(\tau, \omega) \text {, s.t. } u=\tilde{v}\right\}, \quad \forall \tau \in \mathbb{R}, \omega \in \Omega .
$$

In this way, the Hausdorff semi-distance between $\widetilde{\mathcal{A}_{k}}$ and $\mathcal{A}_{\infty}$ lies in the same space $H_{g}\left(\mathcal{O}_{\infty}\right)$ where the Hausdorff semi-distance can be understood in the following sense:

$$
\operatorname{dist}_{H_{g}\left(\mathcal{O}_{\infty}\right)}\left(\widetilde{\mathcal{A}_{k}}(\tau, \omega), \mathcal{A}_{\infty}(\tau, \omega)\right)=\sup _{u \in \widetilde{\mathcal{A}_{k}}(\tau, \omega)} \inf _{v \in \mathcal{A}_{\infty}(\tau, \omega)}\|u-v\|_{H_{g}\left(\mathcal{O}_{\infty}\right)} .
$$

Then our aim is to prove

$$
\operatorname{dist}_{H_{g}\left(\mathcal{O}_{\infty}\right)}\left(\widetilde{\mathcal{A}_{k}}(\tau, \omega), \mathcal{A}_{\infty}(\tau, \omega)\right) \rightarrow 0 \quad \text { as } k \rightarrow \infty
$$

However, the usual energy equation method is not sufficient to prove the large-domain stability from $\mathcal{A}_{k}$ to $\mathcal{A}_{\infty}$ as $k \rightarrow \infty$. We will expand each cocycle $\Phi_{k}\left(\right.$ on $H_{g}\left(\mathcal{O}_{k}\right)$ ) to the null-expansion cocycle $\widetilde{\Phi}_{k}$ on a subspace of $H_{g}\left(\mathcal{O}_{\infty}\right)$ and prove that the sequence of expanding cocycles $\left\{\widetilde{\Phi}_{k}\right\}_{k}$ is equi-asymptotically compact (uniformly in $k$ ) in $H_{g}\left(\mathcal{O}_{\infty}\right)$.

For this end, we develop the usual energy equation method from a single system to a sequence of systems, and prove weak equi-continuity of expanding cocycles $\left\{\widetilde{\Phi}_{k}\right\}_{k}$, which together with an energy equality can help us to establish the equi-asymptotic compactness of expanding cocycles $\left\{\widetilde{\Phi}_{k}\right\}_{k}$.

Finally, by proving the convergence from $\widetilde{\Phi}_{k}$ to $\Phi_{\infty}$ in $H_{g}\left(\mathcal{O}_{\infty}\right)$, we establish the largedomain stability (5) as desired.

This paper is organized as follows. In the next section, the functional spaces and a continuous cocycle for the stochastic $g$-Navier-Stokes equations are defined. In Sect. 3, we define the expanding cocycles and prove the convergence of the expanding cocycles for stochastic $g$-Navier-Stokes equations. We derive the uniform estimates and weak equicontinuity of the solution sequence $\left\{v_{k}\right\}$ in Sect. 4 , which yields the equi-asymptotic compactness of the sequence $\left\{\widetilde{\Phi}_{k}\right\}_{k}$ of expanding cocycles in Sect. 5. In the last section, we show the existence and large-domain stability of the attractor when the domain changes from bounded to unbounded.

\section{Random attractor on varying domain}

\subsection{Functional spaces and operators}

As pointed out in Sect. 1, the unbounded domain $\mathcal{O}_{\infty}$ is of Poincaré type, and thus there exists $\lambda_{\infty}>0$ such that

$$
\int_{\mathcal{O}_{\infty}}|\nabla \zeta(x)|^{2} d x \geq \lambda_{\infty} \int_{\mathcal{O}_{\infty}}|\zeta(x)|^{2} d x, \quad \forall \zeta \in H_{0}^{1}\left(\mathcal{O}_{\infty}\right)
$$

Let $\mathcal{O}_{k}=\left\{x \in \mathcal{O}_{\infty}:|x| \leq k\right\}$, and for each $k \in \overline{\mathbb{N}}$, we use $\left(L_{g}^{2}\left(\mathcal{O}_{k}\right),\|\cdot\|_{g}\right)$ to denote the space $L^{2}\left(\mathcal{O}_{k}\right)$ with the following norm:

$$
\|\zeta\|_{g}^{2}:=\int_{\mathcal{O}_{k}} g(x)|\zeta(x)|^{2} d x, \quad \forall \zeta \in L^{2}\left(\mathcal{O}_{k}\right), k \in \overline{\mathbb{N}}
$$


By (3), one can show that $m_{0}\|\zeta\|^{2} \leq\|\zeta\|_{g}^{2} \leq M_{0}\|\zeta\|^{2}$. So, both norms $\|\cdot\|_{g}$ and $\|\cdot\|$ are indeed equivalent.

Also, we use $\left(H_{0, g}^{1}\left(\mathcal{O}_{k}\right),\|\cdot\|_{H_{0, g}^{1}}\right)$ to denote the space $H_{0}^{1}\left(\mathcal{O}_{k}\right)$ with the following norm:

$$
\|\zeta\|_{H_{0, g}^{1}\left(\mathcal{O}_{k}\right)}^{2}:=\int_{\mathcal{O}_{k}} g(x)|\nabla \zeta(x)|^{2} d x, \quad \forall \zeta \in H_{0}^{1}\left(\mathcal{O}_{k}\right), k \in \overline{\mathbb{N}}
$$

Then, by [16], there exists $\lambda_{0}>0$ (independent of $k$ ) such that

$$
\|\zeta\|_{H_{0, g}^{1}\left(\mathcal{O}_{k}\right)}^{2} \geq \lambda_{0}\|\zeta\|_{g}^{2}, \quad \forall \zeta \in H_{0}^{1}\left(\mathcal{O}_{k}\right), k \in \overline{\mathbb{N}}
$$

which implies that the new norm is (uniformly) equivalent to the original $H_{0}^{1}\left(\mathcal{O}_{k}\right)$-norm.

To reformulate system (2), we introduce some function space:

$$
\begin{aligned}
& \mathcal{V}\left(\mathcal{O}_{k}\right)=\left\{u \in \mathbb{C}_{0}^{\infty}\left(\mathcal{O}_{k}\right): \nabla \cdot(g u)=0\right\}, \\
& H_{g}\left(\mathcal{O}_{k}\right)=\operatorname{cl}_{\mathbb{L}_{g}^{2}\left(\mathcal{O}_{k}\right)} \mathcal{V}, \quad V_{g}\left(\mathcal{O}_{k}\right)=\operatorname{cl}_{\mathbb{H}_{0, g}^{1}\left(\mathcal{O}_{k}\right)} \mathcal{V},
\end{aligned}
$$

where $\mathrm{cl}_{X}$ denotes the closure taken in $X$ and $\mathbb{C}_{0}^{\infty}\left(\mathcal{O}_{k}\right)=C_{0}^{\infty}\left(\mathcal{O}_{k}\right)^{2}, \mathbb{L}_{g}^{2}\left(\mathcal{O}_{k}\right):=L_{g}^{2}\left(\mathcal{O}_{k}\right)^{2}$, $\mathbb{H}_{0, g}^{1}\left(\mathcal{O}_{k}\right)=H_{0, g}^{1}\left(\mathcal{O}_{k}\right)^{2}$, respectively.

Then $H_{g}\left(\mathcal{O}_{k}\right), V_{g}\left(\mathcal{O}_{k}\right)$ are Hilbert spaces with the inner products $(\cdot, \cdot)_{g}$ and $((\cdot, \cdot))_{g}$ of $\mathbb{L}_{g}^{2}\left(\mathcal{O}_{k}\right)$ given by, for $u=\left(u_{1}, u_{2}\right), v=\left(v_{1}, v_{2}\right) \in H_{g}\left(\mathcal{O}_{k}\right)$,

$$
(u, v)_{g}=\int_{\mathcal{O}_{k}} u \cdot v g d x=\sum_{i=1}^{2}\left(u_{i}, v_{i}\right)_{g} \quad \text { and } \quad\|u\|_{g}=(u, u)_{g}^{1 / 2}
$$

and for all $u=\left(u_{1}, u_{2}\right), v=\left(v_{1}, v_{2}\right) \in V_{g}\left(\mathcal{O}_{k}\right)$,

$$
((u, v))_{g}:=(D u, D v)_{g}=\sum_{i, j=1}^{2} \int_{\mathcal{O}_{k}} \frac{\partial u_{j}}{\partial x_{i}} \frac{\partial v_{j}}{\partial x_{i}} g d x \text { and }\|u\|_{V_{g}}=(D u, D u)_{g}^{1 / 2}
$$

where $D u=\left(\left(\frac{\partial u_{1}}{\partial x_{1}}, \frac{\partial u_{1}}{\partial x_{2}}\right),\left(\frac{\partial u_{2}}{\partial x_{1}}, \frac{\partial u_{2}}{\partial x_{2}}\right)\right)$.

Now, we can define the $g$-Laplace operator as follows:

$$
-\Delta_{g} u=-\frac{1}{g}(\nabla \cdot g \nabla) u=-\Delta u-\frac{1}{g}(\nabla g \cdot \nabla) u .
$$

Then, the first equation of (2) can be rewritten as

$$
d u_{k}-\left(v \Delta_{g} u_{k}-\frac{v}{g}(\nabla g \cdot \nabla) u_{k}-\left(u_{k} \cdot \nabla\right) u_{k}-\nabla p\right) d t=f(x, t) d t+\varepsilon h(x) d W(t) .
$$

Consider the $g$-orthogonal projection $P_{g, k}: \mathbb{L}_{g}^{2}\left(\mathcal{O}_{k}\right) \rightarrow H_{g}\left(\mathcal{O}_{k}\right)$ and define the $g$-Stokes operator[18] by

$$
A_{g, k} u=-P_{g, k}\left(\frac{1}{g}(\nabla \cdot g \nabla) u\right), \quad\left\langle A_{g, k} u, v\right\rangle_{g}=\left\langle A_{g, k} u, g v\right\rangle=((u, v))_{g}
$$


for all $u, v \in V_{g}\left(\mathcal{O}_{k}\right)$. Let $V_{g}^{*}\left(\mathcal{O}_{k}\right)$ be the dual space of $V_{g}\left(\mathcal{O}_{k}\right)$. Then $A_{g, k}: V_{g}\left(\mathcal{O}_{k}\right) \rightarrow V_{g}^{*}\left(\mathcal{O}_{k}\right)$ is a homomorphism with $\left\|A_{g, k}\right\| \leq 1 / m_{0}$ (see [16]) and the bound is independent of $k \in \overline{\mathbb{N}}$.

Furthermore, we consider the uniform bound of operator $R_{g, k}: V_{g}\left(\mathcal{O}_{k}\right) \rightarrow V_{g}^{*}\left(\mathcal{O}_{k}\right)$ given by

$$
R_{g, k} u=P_{g, k}\left(\frac{1}{g}(\nabla g \cdot \nabla) u\right), \quad \forall u \in V_{g}\left(\mathcal{O}_{k}\right) .
$$

In this case, by [16], we have the following result.

Lemma 2.1 For each $k \in \overline{\mathbb{N}}$ and $u \in V_{g}\left(\mathcal{O}_{k}\right)$, we have the following uniform bounds:

$$
\|u\|_{H_{g}\left(\mathcal{O}_{k}\right)}^{2} \leq \frac{1}{\lambda_{0}}\|u\|_{V_{g}\left(\mathcal{O}_{k}\right)}^{2} \quad \text { and } \quad\left\|R_{g, k} u\right\|_{V_{g}^{*}\left(\mathcal{O}_{k}\right)} \leq \frac{\|\nabla g\|_{\infty}}{m_{0}^{2} \lambda_{0}^{1 / 2}}\|u\|_{V_{g}\left(\mathcal{O}_{k}\right)}
$$

where $\lambda_{0}$ is given by (7) and $\|\cdot\|_{\infty}$ is the norm in $L^{\infty}\left(\mathcal{O}_{\infty}\right)$.

In the sequel, we will define the bilinear operator $B_{g, k}: V_{g}\left(\mathcal{O}_{k}\right) \times V_{g}\left(\mathcal{O}_{k}\right) \rightarrow V_{g}^{*}\left(\mathcal{O}_{k}\right)$ and the trilinear form $b_{g, k}: V_{g}\left(\mathcal{O}_{k}\right) \times V_{g}\left(\mathcal{O}_{k}\right) \times V_{g}\left(\mathcal{O}_{k}\right) \rightarrow \mathbb{R}$ by

$$
\left\langle B_{g, k}(u, v), w\right\rangle_{g}=b_{g, k}(u, v, w)=\sum_{i, j=1}^{2} \int_{\mathcal{O}_{k}} u_{i} \frac{\partial v_{j}}{\partial x_{i}} w_{j} g d x, \quad \forall u, v, w \in V_{g}\left(\mathcal{O}_{k}\right)
$$

and we write $B_{g, k}(u)=B_{g, k}(u, u)$ without confusion. By Roh [18], we have

$$
\begin{aligned}
& b_{g, k}(u, v, v)=0, \quad b_{g, k}(u, v, w)=-b_{g, k}(u, w, v), \\
& \left\|B_{g, k}(u)\right\|_{V_{g}^{*}} \leq c\|u\|_{g}\|u\|_{V_{g}}, \\
& \left|b_{g, k}(u, v, w)\right| \leq c\|u\|_{g}^{\frac{1}{2}}\|u\|_{V_{g}}^{\frac{1}{2}}\|v\|_{V_{g}}\|w\|_{g}^{\frac{1}{2}}\|w\|_{V_{g}}^{\frac{1}{2}} .
\end{aligned}
$$

Therefore, we can rewrite (8) in the sense of abstract equation

$$
d u_{k}+\left(v A_{g, k} u_{k}+B_{g, k}\left(u_{k}, u_{k}\right)+v R_{g, k} u_{k}\right) d t=\left.P_{g, k} f(x, t)\right|_{\mathcal{O}_{k}} d t+\varepsilon h(x) d W(t) .
$$

Definition 2.2 For a function $u: \mathcal{O}_{k} \rightarrow \mathbb{R}^{2}$, its null-expansion $\tilde{u}: \mathcal{O}_{\infty} \rightarrow \mathbb{R}^{2}$ is defined by

$$
\tilde{u}(x)= \begin{cases}u(x) & \text { for } x \in \mathcal{O}_{k} \\ 0 & \text { for } x \in \mathcal{O}_{\infty} \backslash \mathcal{O}_{k}\end{cases}
$$

Conversely, for a function $v: \mathcal{O}_{\infty} \rightarrow \mathbb{R}^{2}$, the restriction $\left.v\right|_{\mathcal{O}_{k}}: \mathcal{O}_{k} \rightarrow \mathbb{R}^{2}$ is given by

$$
\left.v\right|_{\mathcal{O}_{k}}(x)=v(x), \quad x \in \mathcal{O}_{k} .
$$

We need to estimate the norms of both expansion and restriction in $H_{g}, V_{g}$, and $V_{g}^{*}$.

Lemma $2.3([16])$

(1) If $u \in H_{g}\left(\mathcal{O}_{k}\right)$, then $\tilde{u} \in H_{g}\left(\mathcal{O}_{\infty}\right)$ and $\|\tilde{u}\|_{H_{g}\left(\mathcal{O}_{\infty}\right)}=\|u\|_{H_{g}\left(\mathcal{O}_{k}\right)}$. 
(2) If $u \in V_{g}\left(\mathcal{O}_{k}\right)$, then $\tilde{u} \in V_{g}\left(\mathcal{O}_{\infty}\right)$ and $\|\tilde{u}\|_{V_{g}\left(\mathcal{O}_{\infty}\right)}=\|u\|_{V_{g}\left(\mathcal{O}_{k}\right)}$.

(3) If $v \in H_{g}\left(\mathcal{O}_{\infty}\right)$, then $v_{\mid \mathcal{O}_{k}} \in H_{g}\left(\mathcal{O}_{k}\right)$ and $\left\|\left.v\right|_{\mathcal{O}_{k}}\right\|_{H_{g}\left(\mathcal{O}_{k}\right)} \leq\|v\|_{H_{g}\left(\mathcal{O}_{\infty}\right)}$.

(4) If $w \in V_{g}^{*}\left(\mathcal{O}_{\infty}\right)$, then the restriction $\left.w\right|_{\mathcal{O}_{k}} \in V_{g}^{*}\left(\mathcal{O}_{\infty}\right)$ and $\left\|\left.w\right|_{\mathcal{O}_{k}}\right\|_{V_{g}^{*}\left(\mathcal{O}_{k}\right)} \leq\|w\|_{V_{g}^{*}\left(\mathcal{O}_{\infty}\right)}$.

\subsection{Cocycles for stochastic $g$-NS equations}

The standard probability space $(\Omega, \mathcal{F}, P)$ will be used in this paper where

$$
\Omega=\{\omega \in C(\mathbb{R}, \mathbb{R}): \omega(0)=0\} \text { and } \lim _{t \rightarrow \pm \infty} \frac{\omega(t)}{t}=0,
$$

$\mathcal{F}$ is the Borel algebra induced by the compact-open topology of $\Omega$, and $P$ is the Wiener measure on $(\Omega, \mathcal{F})$. Given $t \in \mathbb{R}$, define $\theta_{t}: \Omega \rightarrow \Omega$ by

$$
\theta_{t} \omega(\cdot)=\omega(\cdot+t)-\omega(t), \quad(\omega, t) \in \Omega \times \mathbb{R} .
$$

Then $\left(\Omega, \mathcal{F}, P,\left\{\theta_{t}\right\}_{t \in \mathbb{R}}\right)$ is a parametric dynamical system. Let $z\left(\theta_{t} \omega\right)=-\int_{-\infty}^{0} e^{s}\left(\theta_{t} \omega\right)(s) d s$, which solves the stochastic Ornstein-Uhlenbeck equation $d z+z d t=d W(t)$. It follows from [1] that there exists a $\theta$-invariant subset $\tilde{\Omega} \subset \Omega$ of full measure such that $z\left(\theta_{t} \omega\right)$ is continuous in $t$ for every $\omega \in \tilde{\Omega}$, and we have the following limits:

$$
\begin{aligned}
& \lim _{t \rightarrow \pm \infty} \frac{z\left(\theta_{t} \omega\right)}{t}=\lim _{t \rightarrow \pm \infty} \frac{1}{t} \int_{-t}^{0} z\left(\theta_{s} \omega\right) d s=\mathbb{E}(z(\omega))=0, \\
& \lim _{t \rightarrow \infty} \frac{1}{t} \int_{-t}^{0}\left|z\left(\theta_{s} \omega\right)\right|^{m} d s=\mathbb{E}\left(|z(\omega)|^{m}\right)=\frac{\Gamma\left(\frac{1+m}{2}\right)}{\sqrt{\pi}}, \quad \forall m>0,
\end{aligned}
$$

where $\mathbb{E}, \Gamma$ denote expectation and gamma function, respectively.

Suppose $h \in H_{g}\left(\mathcal{O}_{\infty}\right)$, then denote by $h_{k}(x):=\left.P_{g, k} h\right|_{\mathcal{O}_{k}}(x)$ for $x \in \mathcal{O}_{k}, k \in \overline{\mathbb{N}}$ and consider the change of variables:

$$
v_{k}\left(t, \tau, \omega, v_{\tau, k}\right)=u_{k}\left(t, \tau, \omega, u_{\tau, k}\right)-\varepsilon z\left(\theta_{t} \omega\right) h_{k},
$$

with $v_{\tau, k}=u_{\tau, k}-\varepsilon z\left(\theta_{\tau} \omega\right) h_{k}$, where we understand $h_{\infty}=P_{g, \infty} h=h$.

In this case, system (13) can be rewritten as follows:

$$
\begin{aligned}
\frac{d v_{k}}{d t} & +v A_{g, k} v_{k}+B_{g, k}\left(v_{k}+\varepsilon h_{k} z\left(\theta_{t} \omega\right)\right)+v R_{g, k} v_{k} \\
& =-\varepsilon v z\left(\theta_{t} \omega\right) A_{g, k} h_{k}-\varepsilon v z\left(\theta_{t} \omega\right) R_{g, k} h_{k}+\left.P_{g, k} f(t)\right|_{\mathcal{O}_{k}}+\varepsilon z\left(\theta_{t} \omega\right) h_{k}
\end{aligned}
$$

with the initial value

$$
v_{k}\left(\tau, \tau, \omega, v_{\tau, k}\right)=v_{\tau, k}=u_{\tau, k}-\varepsilon z\left(\theta_{\tau} \omega\right) h_{k} .
$$

Definition 2.4 The function $v_{k}\left(\cdot, \tau, \omega, v_{\tau, k}\right)$ is called a weak solution of Eqs. (18)-(19) if

$$
v_{k} \in C\left([\tau, \infty), H_{g}\left(\mathcal{O}_{k}\right)\right) \cap L_{\mathrm{loc}}^{2}\left((\tau, \infty), V_{g}\left(\mathcal{O}_{k}\right)\right)
$$


and, for all $t \geq \tau, P$-a.s. $\omega \in \Omega$, and $w \in V_{g}\left(\mathcal{O}_{k}\right)$,

$$
\begin{aligned}
& \left(v_{k}(t), w\right)_{g}+v \int_{\tau}^{t}\left\langle A_{g, k} v_{k}, w\right\rangle_{g} d s+v \int_{\tau}^{t}\left\langle R_{g, k} v_{k}, g w\right\rangle d s \\
& \quad+\int_{\tau}^{t}\left\langle B_{g, k}\left(v_{k}+\varepsilon h_{k} z\left(\theta_{s} \omega\right)\right), w\right\rangle_{g} d s+\varepsilon v \int_{\tau}^{t} z\left(\theta_{s} \omega\right)\left\langle A_{g, k} h_{k}+R_{g, k} h_{k}, w\right\rangle_{g} d s \\
& =\left(v_{\tau, k}, w\right)_{g}+\int_{\tau}^{t}\left\langle\left. f(s)\right|_{\mathcal{O}_{k}}+\varepsilon h_{k} z\left(\theta_{s} \omega\right), g w\right\rangle d s .
\end{aligned}
$$

Lemma 2.5 Assume that $f \in L_{\mathrm{loc}}^{2}\left(\mathbb{R}, V_{g}^{*}\left(\mathcal{O}_{\infty}\right)\right)$. Then, for each $k \in \overline{\mathbb{N}}$ and $v_{\tau, k} \in H_{g}\left(\mathcal{O}_{k}\right)$, system (18)-(19) has a unique weak solution $v_{k}$ in the sense of Definition 2.4. Moreover, the solution $v_{k}\left(t, \tau, \omega ; v_{\tau, k}\right)$ is continuous in $v_{\tau, k}$ and measurable in $\omega$.

Then we can define a family of measurable mappings $\Phi_{k}: \mathbb{R}^{+} \times \mathbb{R} \times \Omega \times H_{g}\left(\mathcal{O}_{k}\right) \rightarrow$ $H_{g}\left(\mathcal{O}_{k}\right)$ corresponding to system (18). Given $\tau \in \mathbb{R}, \omega \in \Omega$, and $v_{\tau, k} \in H_{g}\left(\mathcal{O}_{k}\right)$, we have

$$
\Phi_{k}(t, \tau, \omega) v_{\tau, k}=v_{k}\left(t+\tau, \tau, \theta_{-\tau} \omega, v_{\tau, k}\right)
$$

where $t \geq 0$. Then, for each $k \in \overline{\mathbb{N}}, \Phi_{k}$ is a continuous cocycle [21] and we have

$$
\Phi_{k}(0, \tau, \omega)=I, \quad \Phi_{k}(t+s, \tau, \omega)=\Phi_{k}\left(t, \tau+s, \theta_{s} \omega\right) \Phi_{k}(s, \tau, \omega)
$$

for all $t, s \geq 0, \tau \in \mathbb{R}$, and $\omega \in \Omega$.

We now take a universe $\mathfrak{D}$ on $H_{g}\left(\mathcal{O}_{\infty}\right)$, which consists of all set-valued mappings $D$ : $\mathbb{R} \times \Omega \rightarrow 2^{H_{g}\left(\mathcal{O}_{\infty}\right)} \backslash\{\emptyset\}$ satisfying

$$
\lim _{t \rightarrow+\infty} e^{-\frac{3}{2} \lambda t}\left\|D\left(\tau-t, \theta_{-t} \omega\right)\right\|_{H_{g}\left(\mathcal{O}_{\infty}\right)}^{2}=0, \quad \tau \in \mathbb{R}, \omega \in \Omega,
$$

where $\|D\|$ denotes the supremum of norms of all elements and $\lambda=\frac{1}{3} \lambda_{0} \nu$.

Denote $\left.\mathfrak{D}\right|_{\mathcal{O}_{k}}$ to be the restriction universe of $\mathfrak{D}$ on $H_{g}\left(\mathcal{O}_{k}\right)$. By Lemma 2.3, we have $\left.D_{k} \in \mathfrak{D}\right|_{\mathcal{O}_{k}}$ if and only if

$$
\lim _{t \rightarrow+\infty} e^{-\frac{3}{2} \lambda t}\left\|D_{k}\left(\tau-t, \theta_{-t} \omega\right)\right\|_{H_{g}\left(\mathcal{O}_{k}\right)}^{2}=0 \quad \text { for } k \in \mathbb{N} .
$$

It is easy to check that if $\left.D_{k} \in \mathfrak{D}\right|_{\mathcal{O}_{k}}$, then $\widetilde{D_{k}} \in \mathfrak{D}$. Furthermore, $\widetilde{\left.D\right|_{\mathcal{O}_{k}}} \neq D$ for $D \in \mathfrak{D}$.

In order to obtain the $\left.\mathfrak{D}\right|_{\mathcal{O}_{k}}$-pullback attractor $\mathcal{A}_{k}$ for all $k \in \overline{\mathbb{N}}$, we make further assumptions.

Assumption G We further assume $g \in W^{1, \infty}\left(\mathcal{O}_{k}\right)$ and

$$
\|\nabla g\|_{\infty} \leq \frac{1}{4} m_{0} \lambda_{0}^{\frac{1}{2}}, \quad \forall k \in \overline{\mathbb{N}}
$$

where $\lambda_{0}$ is given by (7).

Assumption $\mathbf{H}$ We take the function $h(x) \in\left(W^{1, \infty}\left(\mathcal{O}_{k}\right)\right)^{2} \cap V_{g}\left(\mathcal{O}_{k}\right) \cap \mathbb{H}^{2}\left(\mathcal{O}_{k}\right), \forall k \in \overline{\mathbb{N}}$. 
Assumption $\mathbf{F} f \in L_{\text {loc }}^{2}\left(\mathbb{R}, V_{g}^{*}\left(\mathcal{O}_{\infty}\right)\right)$, we assume that, for $\lambda=\frac{1}{3} \lambda_{0} \nu$,

$$
\int_{-\infty}^{0} e^{\lambda s}\|f(s+\tau)\|_{V_{g}^{*}\left(\mathcal{O}_{\infty}\right)}^{2} d s<\infty, \quad \forall \tau \in \mathbb{R}
$$

By Lemma 2.3(4) and Assumption F, the restriction $\left.f\right|_{\mathcal{O}_{k}}$ is still tempered:

$$
\int_{-\infty}^{0} e^{\lambda s}\left\|\left.f\right|_{\mathcal{O}_{k}}(s+\tau)\right\|_{V_{g}^{*}\left(\mathcal{O}_{k}\right)}^{2} d s<\infty, \quad \forall \tau \in \mathbb{R}, k \in \mathbb{N} .
$$

Assumption S (Small noise) The density of noise $\varepsilon \in\left(0, \varepsilon_{0}\right]$ is small enough, where

$$
\varepsilon_{0}=\min \left\{\frac{\sqrt{\pi} \lambda}{4 c_{0}}, 1\right\} \quad \text { and } \quad c_{0}=2 M_{0}(\|h\|+1)\|\nabla h\|_{\mathbb{L}^{\infty}\left(\mathcal{O}_{\infty}\right)}
$$

\section{Random attractor and convergence of expanding cocycles}

\subsection{Expanding cocycles}

In this section, we need to expand the cocycle $\Phi_{k}$ from $H_{g}\left(\mathcal{O}_{k}\right)$ to $H_{g}\left(\mathcal{O}_{\infty}\right)$. For this end, we define the corresponding null-expansion of an operator $\Phi_{k}(t, \tau, \omega): H_{g}\left(\mathcal{O}_{k}\right) \rightarrow H_{g}\left(\mathcal{O}_{k}\right)$ by $\widetilde{\Phi_{k}}: H_{g}\left(\mathcal{O}_{\infty}\right) \rightarrow H_{g}\left(\mathcal{O}_{\infty}\right)$,

$$
\left(\widetilde{\Phi_{k}} u\right)(x)=\left(\Phi_{k}\left(\left.u\right|_{\mathcal{O}_{k}}\right)\right)(x), \quad \forall x \in \mathcal{O}_{k}, \quad\left(\widetilde{\Phi_{k}} u\right)(x)=0, \quad \forall x \in \mathcal{O}_{\infty} \backslash \mathcal{O}_{k}
$$

However, in general, $\widetilde{\left.u\right|_{\mathcal{O}_{k}}} \neq u$ for $u \in H_{g}\left(\mathcal{O}_{\infty}\right)$ and so $\widetilde{\Phi_{k}}(0, \tau, \omega)$ is not an identical operator on $H_{g}\left(\mathcal{O}_{\infty}\right)$. Hence, $\widetilde{\Phi}_{k}$ is not a cocycle on the whole space $H_{g}\left(\mathcal{O}_{\infty}\right)$.

Fortunately, we can show that the null-expansion $\widetilde{\Phi}_{k}$ is a cocycle on the closed linear subspace $H_{k}\left(\mathcal{O}_{\infty}\right)$ defined by

$$
H_{k}\left(\mathcal{O}_{\infty}\right):=\left\{u \in H_{g}\left(\mathcal{O}_{\infty}\right): u(x)=0 \text { for } x \in \mathcal{O}_{\infty} \backslash \mathcal{O}_{k}\right\}
$$

and thus $H_{k}\left(\mathcal{O}_{\infty}\right)$ is a Banach space with the same norm as in $H_{g}\left(\mathcal{O}_{\infty}\right)$.

A $\mathfrak{D}$-pullback random attractor means a bi-parametric set which is measurable, compact, invariant, and $\mathfrak{D}$-pullback attracting. For the concept and existence theorem, the reader can refer to[21].

Theorem 3.1 For each $k \in \overline{\mathbb{N}}$, the null-expansion $\widetilde{\Phi}_{k}$ of $\Phi_{k}$ is a cocycle on $H_{k}\left(\mathcal{O}_{\infty}\right)$ and it has a $\widetilde{\mathfrak{D}_{k}}$-pullback random attractor in $H_{k}\left(\mathcal{O}_{\infty}\right)$, given by the null-expansion $\widetilde{\mathcal{A}_{k}}$ of the random attractor $\mathcal{A}_{k}$.

Proof By (27), for each $u \in H_{k}\left(\mathcal{O}_{\infty}\right)$, we have

$$
\widetilde{\Phi_{k}}(0, \tau, \omega) u=\text { expansion of }\left.\Phi_{k}(0, \tau, \omega) u\right|_{\mathcal{O}_{k}}=\widetilde{\left.u\right|_{\mathcal{O}_{k}}}=u
$$

So, $\widetilde{\Phi_{k}}(0, \tau, \omega)$ is the identical operator on $H_{k}\left(\mathcal{O}_{\infty}\right)$. Other properties of cocycle are easily verified.

Next, we prove that $\widetilde{\mathcal{A}_{k}}$ is a $\widetilde{\mathfrak{D}_{k}}$-pullback random attractor for $\widetilde{\Phi_{k}}$ in four steps. 
Step 1. We show that $\widetilde{\mathcal{A}_{k}}$ is measurable on $H_{k}\left(\mathcal{O}_{\infty}\right)$. Let $u \in H_{k}\left(\mathcal{O}_{\infty}\right)$, which means $u \equiv 0$ on $\mathcal{O}_{\infty} \backslash \mathcal{O}_{k}$ and thus

$$
\|u\|_{H_{k}\left(\mathcal{O}_{\infty}\right)}^{2}=\int_{\mathcal{O}_{\infty}}|u(x)|^{2} g d x=\int_{\mathcal{O}_{k}}|u(x)|^{2} g d x=\left\|\left.u\right|_{\mathcal{O}_{k}}\right\|_{H_{g}\left(\mathcal{O}_{k}\right)}^{2} .
$$

This equality implies that

$$
d_{H_{k}\left(\mathcal{O}_{\infty}\right)}\left(u, \widetilde{\mathcal{A}_{k}}(\tau, \omega)\right)=d_{H_{g}\left(\mathcal{O}_{k}\right)}\left(\left.u\right|_{\mathcal{O}_{k}}, \mathcal{A}_{k}(\tau, \omega)\right) \text {. }
$$

Since $\mathcal{A}_{k}(\tau, \omega)$ is measurable on $H_{g}\left(\mathcal{O}_{k}\right)$, the above equality implies the measurability of the mapping $\omega \rightarrow d_{H_{k}\left(\mathcal{O}_{\infty}\right)}\left(u, \widetilde{\mathcal{A}}_{k}(\tau, \omega)\right)$ for each $u \in H_{k}\left(\mathcal{O}_{\infty}\right)$, that is, $\widetilde{\mathcal{A}_{k}}$ is measurable on $H_{k}\left(\mathcal{O}_{\infty}\right)$.

Step 2. We show that $\widetilde{\mathcal{A}_{k}}(\tau, \omega)$ is a compact set in $H_{k}\left(\mathcal{O}_{\infty}\right)$. Let $\left\{u_{n}\right\}$ be a sequence of $\widetilde{\mathcal{A}_{k}}(\tau, \omega)$. Then there exists $v_{n} \in \mathcal{A}_{k}(\tau, \omega)$ such that $u_{n}=\widetilde{v_{n}}$. Due to $\mathcal{A}_{k}(\tau, \omega)$ is compact in $H_{g}\left(\mathcal{O}_{k}\right)$, passing to a subsequence, we have $v_{n^{\prime}} \rightarrow v \in \mathcal{A}_{k}(\tau, \omega)$. Denote $\widetilde{v}$ by the nullexpansion of $v$, then $\widetilde{v} \in \widetilde{\mathcal{A}}_{k}$. By Lemma 2.3(1),

$$
\left\|u_{n^{\prime}}-\widetilde{v}\right\|_{H_{g}\left(\mathcal{O}_{\infty}\right)}=\left\|\widetilde{v_{n^{\prime}}}-\widetilde{v}\right\|_{H_{g}\left(\mathcal{O}_{\infty}\right)}=\left\|v_{n^{\prime}}-v\right\|_{H_{g}\left(\mathcal{O}_{k}\right)} \rightarrow 0 \quad \text { as } n^{\prime} \rightarrow \infty
$$

which implies that $u_{n^{\prime}} \rightarrow \widetilde{v} \in \widetilde{\mathcal{A}_{k}}(\tau, \omega)$. Therefore, $\widetilde{\mathcal{A}_{k}}(\tau, \omega)$ is compact in $H_{k}\left(\mathcal{O}_{\infty}\right)$.

Step 3. We show the invariance. Given $u \in \widetilde{\mathcal{A}_{k}}\left(t+\tau, \theta_{t} \omega\right)$, there is $v \in \mathcal{A}_{k}\left(t+\tau, \theta_{t} \omega\right)$ such that the null-expansion $\widetilde{v}=u$. By the negative invariance of $\mathcal{A}_{k}(\tau, \omega)$, there is $w \in \mathcal{A}_{k}(\tau, \omega)$ such that

$$
v=\Phi_{k}(t, \tau, \omega) w
$$

Let $\widetilde{w}$ be the null-expansion of $w$, then $\widetilde{w}_{\mid \mathcal{O}_{k}}=w$. It follows from (27) that we have

$$
u=\widetilde{v}=\widetilde{\Phi}_{k}(t, \tau, \omega) \widetilde{w} \in \widetilde{\Phi}_{k}(t, \tau, \omega) \widetilde{\mathcal{A}_{k}}(\tau, \omega)
$$

which proves the negative invariance of $\widetilde{\mathcal{A}_{k}}$. Similarly, one can prove the positive invariance.

Step 4. We show that $\widetilde{\mathcal{A}_{k}}$ is $\widetilde{\mathfrak{D}_{k}}$-pullback attracting. Let $D \in \widetilde{\mathfrak{D}_{k}}$, then there is $D_{k} \in \mathfrak{D}_{k}$ such that $D=\widetilde{D_{k}}$. By the same method as given in (28), we know that

$$
\begin{aligned}
& \operatorname{dist}_{H_{k}\left(\mathcal{O}_{\infty}\right)}\left(\widetilde{\Phi}_{k}\left(t, \tau-t, \theta_{-t} \omega\right) D\left(\tau-t, \theta_{-t} \omega\right), \widetilde{\mathcal{A}}_{k}(\tau, \omega)\right) \\
& \quad=\operatorname{dist}_{H_{g}\left(\mathcal{O}_{k}\right)}\left(\Phi_{k}\left(t, \tau-t, \theta_{-t} \omega\right) D_{k}\left(\tau-t, \theta_{-t} \omega\right), \mathcal{A}_{k}(\tau, \omega)\right) .
\end{aligned}
$$

Then the attraction of $\widetilde{\mathcal{A}_{k}}$ follows from the attraction of $\mathcal{A}_{k}$.

\subsection{Convergence of expanding cocycles}

In this subsection, we prove the convergence of the expanding cocycles as follows.

Lemma 3.2 Let $v_{0, k} \in H_{g}\left(\mathcal{O}_{k}\right)(k \in \overline{\mathbb{N}})$ such that $\left\|\widetilde{v_{0, k}}-v_{0, \infty}\right\|_{H_{g}\left(\mathcal{O}_{\infty}\right)} \rightarrow 0$ as $k \rightarrow \infty$. Then

$$
\left\|{\widetilde{v_{k}}}_{(}\left(t, \tau, \omega ; v_{0, k}\right)-v_{\infty}\left(t, \tau, \omega ; v_{0, \infty}\right)\right\|_{H_{g}\left(\mathcal{O}_{\infty}\right)} \rightarrow 0, \quad \text { as } k \rightarrow \infty
$$

uniformly in $t \in[\tau, \tau+T]$ for any $T>0$. 
Proof By considering the expansion, we rewrite Eq. (18) on the domain $\mathcal{O}_{\infty}$ for all $t \in$ $[\tau, \tau+T], k \in \mathbb{N}:$

$$
\begin{aligned}
& \frac{d \widetilde{v_{k}}}{d t}+\nu A_{g, \infty} \widetilde{v_{k}}+\varepsilon v z\left(\theta_{t} \omega\right) A_{g, \infty} \widetilde{h_{k}}+B_{g, \infty}\left(\widetilde{v_{k}}+\varepsilon \widetilde{h_{k}} z\left(\theta_{t} \omega\right)\right) \\
& +v R_{g, \infty} \widetilde{v_{k}}+\varepsilon v z\left(\theta_{t} \omega\right) R_{g, \infty} \widetilde{h_{k}}=\widetilde{\left.f(t)\right|_{\mathcal{O}_{k}}}+\varepsilon z\left(\theta_{t} \omega\right) \tilde{h_{k}},
\end{aligned}
$$

where $\widetilde{v_{\infty}}=v_{\infty}$ and $\widetilde{\left.f(t)\right|_{\mathcal{O}_{k}}}$ is regarded as the null-expansion of the restriction of $f(t)$.

Let $V_{k}=\widetilde{v_{k}}-v_{\infty} \in H_{g}\left(O_{\infty}\right)$. Subtracting Eq. (18) for $k=\infty$ from (30) and multiplying the result by $g V_{k}$, we have

$$
\begin{aligned}
\frac{d}{d t}\left\|V_{k}\right\|_{g}^{2}+2 v\left\|D V_{k}\right\|_{g}^{2}= & -2 b_{g, \infty}\left(\widetilde{v_{k}}+\varepsilon \tilde{h_{k}} z\left(\theta_{t} \omega\right), \widetilde{v_{k}}+\varepsilon \tilde{h_{k}} z\left(\theta_{t} \omega\right), V_{k}\right) \\
& +2 b_{g, \infty}\left(v_{\infty}+\varepsilon h z\left(\theta_{t} \omega\right), v_{\infty}+\varepsilon h z\left(\theta_{t} \omega\right), V_{k}\right)-2 v\left\langle R_{g, k} V_{k}, g V_{k}\right\rangle \\
& -\varepsilon v z\left(\theta_{t} \omega\right)\left\langle\left(A_{g, \infty}+R_{g, \infty}\right)\left(\widetilde{h_{k}}-h\right), g V_{k}\right\rangle \\
& +2\left(\widetilde{\left.f(t)\right|_{\mathcal{O}_{k}}}-f(t), g V_{k}\right\rangle+\varepsilon z\left(\theta_{t} \omega\right)\left(\widetilde{h_{k}}-h, g V_{k}\right) .
\end{aligned}
$$

By (10) and the trilinear property of $b_{g, \infty}$,

$$
\begin{aligned}
I_{1}:= & -2\left(b_{g, \infty}\left(\widetilde{v_{k}}+\varepsilon \widetilde{h_{k}} z\left(\theta_{t} \omega\right), \widetilde{v_{k}}+\varepsilon \widetilde{h_{k}} z\left(\theta_{t} \omega\right), V_{k}\right)\right. \\
& +2 b_{g, \infty}\left(v_{\infty}+\varepsilon h z\left(\theta_{t} \omega\right), v_{\infty}+\varepsilon h z\left(\theta_{t} \omega\right), V_{k}\right) \\
= & -2 b_{g, \infty}\left(V_{k}+\varepsilon z\left(\theta_{t} \omega\right)\left(\widetilde{h_{k}}-h\right), \widetilde{v_{k}}+\varepsilon \widetilde{h_{k}} z\left(\theta_{t} \omega\right), V_{k}\right) \\
& -2 b_{g, \infty}\left(v_{\infty}+\varepsilon z\left(\theta_{t} \omega\right) h, V_{k}+\varepsilon z\left(\theta_{t} \omega\right)\left(\widetilde{h_{k}}-h\right), V_{k}\right) \\
= & -2 b_{g, \infty}\left(V_{k}+\varepsilon z\left(\theta_{t} \omega\right)\left(\widetilde{h_{k}}-h\right), v_{\infty}+\varepsilon \widetilde{h_{k}} z\left(\theta_{t} \omega\right), V_{k}\right) \\
& -2 b_{g, \infty}\left(v_{\infty}+\varepsilon z\left(\theta_{t} \omega\right) h, \varepsilon z\left(\theta_{t} \omega\right)\left(\widetilde{h_{k}}-h\right), V_{k}\right) .
\end{aligned}
$$

Notice that $\varepsilon z\left(\theta_{t} \omega\right)$ is bounded in $t \in[\tau, \tau+T], \varepsilon \leq \varepsilon_{0}$ and the sequence $\left\{\tilde{h_{k}}: k \in \overline{\mathbb{N}}\right\}$ is bounded in $V_{g}\left(\mathcal{O}_{\infty}\right)$. We infer from (12) and (32) that

$$
\begin{aligned}
I_{1} & \leq 2\left\|V_{k}\right\|_{g}\left\|D V_{k}\right\|_{g}\left(\left\|D v_{\infty}\right\|_{g}+c\right)+c\left\|D\left(\tilde{h_{k}}-h\right)\right\|_{g}\left(\left\|D v_{\infty}\right\|_{g}+c\right)\left\|D V_{k}\right\|_{g} \\
& \leq \frac{v}{2}\left\|D V_{k}\right\|_{g}^{2}+c\left(\left\|D v_{\infty}\right\|_{g}^{2}+1\right)\left\|V_{k}\right\|_{g}^{2}+c\left(\left\|D v_{\infty}\right\|_{g}^{2}+1\right)\left\|D\left(\tilde{h_{k}}-h\right)\right\|_{g}^{2} .
\end{aligned}
$$

By Assumption G,

$$
\begin{aligned}
I_{2} & :=-2 v\left\langle R_{g, k} V_{k}, g V_{k}\right\rangle \leq 2 v\left|\left\langle\left(\frac{\nabla g}{g} \cdot \nabla\right) V_{k}, g V_{k}\right\rangle\right| \\
& \leq \frac{2 v\|\nabla g\|_{\infty}}{m_{0} \lambda_{0}^{1 / 2}}\left\|D V_{k}\right\|_{g}^{2} \leq \frac{v}{2}\left\|D V_{k}\right\|_{g}^{2} .
\end{aligned}
$$

Since $A_{g, \infty}$ and $R_{g, \infty}$ are bounded linear operators from $V_{g}\left(\mathcal{O}_{\infty}\right)$ to $V_{g}^{*}$, we have

$$
\begin{aligned}
I_{3} & :=-\varepsilon v z\left(\theta_{t} \omega\right)\left\langle\left(A_{g, \infty}+R_{g, \infty}\right)\left(\tilde{h_{k}}-h\right), g V_{k}\right\rangle \\
& \leq \frac{v}{2}\left\|D V_{k}\right\|_{g}^{2}+c\left\|D\left(\tilde{h_{k}}-h\right)\right\|_{g}^{2} .
\end{aligned}
$$


For the forcing term, since $V_{k}=\tilde{v_{k}}-v_{\infty}=-v_{\infty}$ on $\mathcal{O}_{\infty} \backslash \mathcal{O}_{k}$, we have

$$
\begin{aligned}
I_{4} & :=2\left|\widehat{\left.f(t)\right|_{\mathcal{O}_{k}}}-f(t), g V_{k}\right\rangle|=2| \int_{\mathcal{O}_{\infty} \backslash \mathcal{O}_{k}} f(t) v_{\infty} g d x \mid \\
& =2\left|\int_{\mathcal{O}_{\infty}} f(t)\left(v_{\infty}-\widetilde{v_{\infty} \mid \mathcal{O}_{k}}\right) g d x\right| \leq 2\|f(t)\|_{V_{g}^{*}\left(\mathcal{O}_{\infty}\right)}\left\|v_{\infty}-\widetilde{v_{\infty} \mid \mathcal{O}_{k}}\right\|_{V_{g}\left(\mathcal{O}_{\infty}\right)} .
\end{aligned}
$$

By the Holder inequality and Poincaré inequality,

$$
I_{5}:=\varepsilon z\left(\theta_{t} \omega\right)\left(\tilde{h_{k}}-h, g V_{k}\right) \leq\left\|V_{k}\right\|_{g}^{2}+c\left\|D\left(\tilde{h_{k}}-h\right)\right\|_{g}^{2}
$$

It follows from (33) to (37) that

$$
\begin{aligned}
\frac{d}{d t}\left\|V_{k}\right\|_{g}^{2} \leq & c\left\|D v_{\infty}\right\|_{g}^{2}\left\|V_{k}\right\|_{g}^{2}+c\|f(t)\|_{V_{g}^{*}\left(\mathcal{O}_{\infty}\right)}\left\|v_{\infty}-\widetilde{\left.v_{\infty}\right|_{\mathcal{O}_{k}}}\right\|_{V_{g}\left(\mathcal{O}_{\infty}\right)} \\
& +c\left(\left\|D v_{\infty}\right\|_{g}^{2}+1\right)\left\|D\left(\tilde{h_{k}}-h\right)\right\|_{g}^{2} .
\end{aligned}
$$

By Gronwall's lemma we get, for all $t \in[\tau, \tau+T]$,

$$
\begin{aligned}
\left\|V_{k}(t)\right\|^{2} \leq & C e^{C \int_{\tau}^{\tau+T}\left\|v_{\infty}(r)\right\|_{V_{g}}^{2} d r}\left(\left\|V_{k}(\tau)\right\|^{2}+\int_{\tau}^{\tau+T}\|f(r)\|_{V_{g}^{*}}\left\|v_{\infty}-\widetilde{v_{\infty} \mid \mathcal{O}_{k}}\right\|_{V_{g}\left(\mathcal{O}_{\infty}\right)} d r\right. \\
& \left.+\left\|D\left(\tilde{h_{k}}-h\right)\right\|_{g}^{2} \int_{\tau}^{\tau+T}\left(\left\|v_{\infty}(r)\right\|_{V_{g}}^{2} d r+1\right) d r\right) .
\end{aligned}
$$

By Lemma 2.5, $v_{\infty} \in L^{2}\left(\tau, \tau+T ; V_{g}\left(\mathcal{O}_{\infty}\right)\right)$ and thus

$$
\int_{\tau}^{\tau+T} \int_{\mathcal{O}_{\infty}}\left|D v_{\infty}(r, x)\right|^{2} d x d r<+\infty
$$

Since $f \in L^{2}\left(\tau, \tau+T ; V_{g}^{*}\left(\mathcal{O}_{\infty}\right)\right)$, it follows from the Holder inequality that, as $k \rightarrow \infty$,

$$
\begin{aligned}
& \int_{\tau}^{\tau+T}\|f(r)\|_{V_{g}^{*}\left(\mathcal{O}_{\infty}\right)}\left\|D\left(v_{\infty}(r)-\widetilde{v_{\infty} \mathcal{O}_{k}}(r)\right)\right\|_{g} d r \\
& \quad \leq\left(\int_{\tau}^{\tau+T}\|f(r)\|_{V_{g}^{*}\left(\mathcal{O}_{\infty}\right)}^{2}\right)^{\frac{1}{2}}\left(\left\|D\left(v_{\infty}(r)-\widehat{v_{\infty_{\mid \mathcal{O}}}}(r)\right)\right\|_{g}^{2} d r\right)^{\frac{1}{2}} \\
& \quad \leq c\left(\int_{\tau}^{\tau+T} \int_{\mathcal{O}_{\infty} \backslash \mathcal{O}_{k}}\left|D v_{\infty}(r, x)\right|^{2} d x d r\right)^{\frac{1}{2}} \rightarrow 0
\end{aligned}
$$

in view of (40) and the Lebesgue controlled convergence theorem. By Assumption $\mathrm{H}$ and the absolute continuity of the integrals, by the convergence of the initial value, we have

$$
\left\|D\left(\tilde{h_{k}}-h\right)\right\|_{g}^{2} \rightarrow 0 \quad \text { as } k \rightarrow \infty
$$

By the assumption that $\left\|V_{k}(\tau)\right\|_{g}^{2} \rightarrow 0$ as $k \rightarrow \infty$, we infer from (39)-(42) that $\left\|V_{k}(t)\right\|_{g}^{2} \rightarrow$ 0 as $k \rightarrow \infty$, uniformly in $t \in[\tau, \tau+T]$. 


\section{Weak equi-continuity of the sequence of expanding cocycles}

\subsection{Uniform absorption}

Lemma 4.1 There is a random variable $C_{0}(\omega)$ such that

$$
c_{0} \varepsilon \int_{-t}^{0}\left|z\left(\theta_{r} \omega\right)\right| d r \leq \frac{1}{2} \lambda t+C_{0}(\omega), \quad \forall t \geq 0, \varepsilon \leq \varepsilon_{0}
$$

Proof By (17) and the ergodic theorem,

$$
\frac{1}{t} \int_{-t}^{0}\left|z\left(\theta_{r} \omega\right)\right| d r \rightarrow \mathbb{E}(|z(\omega)|)=\frac{1}{\sqrt{\pi}} \quad \text { as } t \rightarrow+\infty
$$

We choose large $t_{0}(\omega) \geq 0$ and use Assumption $S$ to obtain, for all $\varepsilon \leq \varepsilon_{0}$,

$$
c_{0} \varepsilon \int_{-t}^{0}\left|z\left(\theta_{r} \omega\right)\right| d r \leq c_{0} \varepsilon_{0} \frac{2}{\sqrt{\pi}} t \leq \frac{1}{2} \lambda t, \quad \forall t \geq t_{0},
$$

while, for all $t \leq t_{0}$,

$$
c_{0} \varepsilon \int_{-t}^{0}\left|z\left(\theta_{r} \omega\right)\right| d r \leq c_{0} \varepsilon_{0} t_{0} \max _{r \in\left[-t_{0}, 0\right]}\left|z\left(\theta_{r} \omega\right)\right|:=C_{0}(\omega)
$$

Let $\mathbb{Q}$ be the set of rational numbers, then $\left[-t_{0}, 0\right] \cap \mathbb{Q}=\left\{r_{1}, r_{2}, \ldots\right\}$ is a countable set. Hence,

$$
\max _{r \in\left[-t_{0}, 0\right]}\left|z\left(\theta_{r} \omega\right)\right|=\sup _{n \in \mathbb{N}}\left|z\left(\theta_{r_{n}} \omega\right)\right|,
$$

which is measurable in $\omega$ and so is $C_{0}(\omega)$. Therefore, (43) holds true.

Lemma 4.2 For each $\tau \in \mathbb{R}, \omega \in \Omega$, and $D \in \mathfrak{D}$, there exists $T=T(\tau, \omega, \sigma, D)>0$ such that, for all $t \geq T, \sigma \in[\tau-t, \tau]$, and $\left.v_{\tau-t, k} \in D\right|_{\mathcal{O}_{k}}\left(\tau-t, \theta_{-t} \omega\right)$,

$$
\sup _{k \in \overline{\mathbb{N}}}\left\|v_{k}\left(\sigma, \tau-t, \theta_{-\tau} \omega, v_{\tau-t, k}\right)\right\|_{H_{g}\left(\mathcal{O}_{k}\right)}^{2} \leq e^{2 \lambda(\tau-\sigma)} \rho(\sigma, \tau, \omega)
$$

where, from Assumptions F, S,

$$
\begin{aligned}
\rho(\sigma, \tau, \omega)= & c \int_{-\infty}^{\sigma-\tau} e^{2 \lambda s+c_{0} \varepsilon \int_{s}^{0}\left|z\left(\theta_{r} \omega\right)\right| d r}\|f(s+\tau)\|_{V_{g}^{*}\left(\mathcal{O}_{\infty}\right)}^{2} d s \\
& +c \int_{-\infty}^{\sigma-\tau} e^{2 \lambda s+c_{0} \varepsilon \int_{s}^{0}\left|z\left(\theta_{r} \omega\right)\right| d r}\left(\left|z\left(\theta_{s} \omega\right)\right|^{2}+\left|z\left(\theta_{s} \omega\right)\right|^{4}\right) d s<+\infty
\end{aligned}
$$

Proof We multiply Eq. (18) by $g v_{k}$ and integrate over $\mathcal{O}_{k}$ to obtain

$$
\begin{aligned}
\frac{d}{d t} & \left\|v_{k}\right\|_{g}^{2}+2 v\left\|v_{k}\right\|_{V_{g}}^{2} \\
= & 2\left\langle\left. f(t)\right|_{\mathcal{O}_{k}}, g v_{k}\right\rangle+2 \varepsilon z\left(\theta_{t} \omega\right)\left(h_{k}, g v_{k}\right)-2 \varepsilon v z\left(\theta_{t} \omega\right)\left\langle A_{g, k} h_{k}, g v_{k}\right\rangle \\
& -2 \varepsilon v z\left(\theta_{t} \omega\right)\left\langle R_{g, k} h_{k}, g v_{k}\right\rangle-2 v\left\langle R_{g, k} v_{k}, g v_{k}\right\rangle-2\left\langle B_{g, k}\left(v_{k}+\varepsilon h_{k} z\left(\theta_{t} \omega\right)\right), v_{k}\right\rangle_{g} .
\end{aligned}
$$


For the nonlinear term, by Lemma 2.3(4), we have

$$
\begin{aligned}
2\left|\left\langle\left. f(t)\right|_{\mathcal{O}_{k}}, g v_{k}\right\rangle\right| & \leq 2 M_{0}\left\|\left.f(t)\right|_{\mathcal{O}_{k}}\right\|_{V_{g}^{*}\left(\mathcal{O}_{k}\right)}\left\|v_{k}\right\|_{V_{g}} \\
& \leq \frac{v}{2}\left\|v_{k}\right\|_{V_{g}}^{2}+\frac{2 M_{0}^{2}}{v}\left\|\left.f(t)\right|_{\mathcal{O}_{k}}\right\|_{V_{g}^{*}\left(\mathcal{O}_{k}\right)}^{2} \\
& \leq \frac{v}{2}\left\|v_{k}\right\|_{V_{g}}^{2}+\frac{2 M_{0}^{2}}{v}\|f(t)\|_{V_{g}^{*}\left(\mathcal{O}_{\infty}\right)}^{2} .
\end{aligned}
$$

By Assumption G and Lemma 2.1, we have

$$
-2 v\left\langle R_{g, k} v_{k}, g v_{k}\right\rangle \leq \frac{2 v\|\nabla g\|_{\infty}}{m_{0}}\left\|v_{k}\right\|_{V_{g}}\left\|v_{k}\right\|_{g} \leq \frac{2 v\|\nabla g\|_{\infty}}{m_{0} \lambda_{0}^{1 / 2}}\left\|v_{k}\right\|_{V_{g}}^{2} .
$$

By Assumption $\mathrm{H}$ and Lemma 2.3,

$$
\begin{aligned}
2 \varepsilon z\left(\theta_{t} \omega\right)\left(h_{k}, g v_{k}\right) & \leq \frac{\lambda}{4}\left\|v_{k}\right\|_{g}^{2}+c\left|z\left(\theta_{t} \omega\right)\right|^{2}\left\|h_{k}\right\|_{H_{g}\left(\mathcal{O}_{k}\right.}^{2} \\
\leq & \frac{\lambda}{4}\left\|v_{k}\right\|_{g}^{2}+c\left|z\left(\theta_{t} \omega\right)\right|^{2}, \\
-2 \varepsilon v z\left(\theta_{t} \omega\right)\left\langle A_{g, k} h_{k}, g v_{k}\right\rangle & \leq 2 v z\left(\theta_{t} \omega\right) \frac{\|\nabla g\|_{\infty}}{m_{0}}\|\nabla h\|_{\mathbb{L}^{\infty}}\left\|v_{k}\right\|_{g} \\
& \leq \frac{\lambda}{4}\left\|v_{k}\right\|_{g}^{2}+c\left|z\left(\theta_{t} \omega\right)\right|^{2}, \\
-2 \varepsilon v z\left(\theta_{t} \omega\right)\left\langle R_{g, k} h_{k}, g v_{k}\right\rangle & \leq 2 v z\left(\theta_{t} \omega\right) \frac{\|\nabla g\|_{\infty}}{m_{0}}\|\nabla h\|_{\mathbb{L}^{\infty}\left\|v_{k}\right\|_{g}} \\
& \leq \frac{\lambda}{4}\left\|v_{k}\right\|_{g}^{2}+c\left|z\left(\theta_{t} \omega\right)\right|^{2} .
\end{aligned}
$$

Using (10), (12), and Assumption H, we have

$$
\begin{aligned}
& 2\left|\left\langle B_{g, k}\left(v_{k}+\varepsilon h_{k} z\left(\theta_{t} \omega\right), v_{k}+\varepsilon h_{k} z\left(\theta_{t} \omega\right)\right), v_{k}\right\rangle_{g}\right| \\
& \quad=2\left|b_{g, k}\left(v_{k}+\varepsilon h_{k} z\left(\theta_{t} \omega\right), \varepsilon h_{k} z\left(\theta_{t} \omega\right), v_{k}\right)\right| \\
& \quad \leq 2 M_{0} \varepsilon\|\nabla h\|_{\mathbb{L}^{\infty}}\left|z\left(\theta_{t} \omega\right)\right|\left\|v_{k}\right\|_{g}^{2}+2 M_{0} \varepsilon^{2}\|h\|\|\nabla h\|_{\mathbb{L}^{\infty}}\left|z\left(\theta_{t} \omega\right)\right|^{2}\left\|v_{k}\right\|_{g} \\
& \quad \leq c_{0} \varepsilon\left(\left|z\left(\theta_{t} \omega\right)\right|\left\|v_{k}\right\|_{g}^{2}+\left|z\left(\theta_{t} \omega\right)\right|^{2}\left\|v_{k}\right\|_{g}\right) \\
& \quad \leq c_{0} \varepsilon\left|z\left(\theta_{t} \omega\right)\right|\left\|v_{k}\right\|_{g}^{2}+\frac{\lambda}{4}\left\|v_{k}\right\|_{g}^{2}+c\left|z\left(\theta_{t} \omega\right)\right|^{4},
\end{aligned}
$$

where $c_{0}=2 M_{0}(\|h\|+1)\|\nabla h\|_{\mathbb{L}^{\infty}}>0$. It follows from (46) to (52) and the Poincaré inequality in (9)

$$
2 v\left\|v_{k}\right\|_{V_{g}}^{2} \geq \lambda_{0} v\left\|v_{k}\right\|_{g}^{2}+v\left\|v_{k}\right\|_{V_{g}}^{2}=3 \lambda\left\|v_{k}\right\|_{g}^{2}+v\left\|v_{k}\right\|_{V_{g}}^{2} .
$$

We obtain that

$$
\begin{gathered}
\frac{d}{d s}\left\|v_{k}\right\|_{g}^{2}+\left(2 \lambda-c_{0} \varepsilon\left|z\left(\theta_{s} \omega\right)\right|\right)\left\|v_{k}\right\|_{g}^{2}+C_{g}\left\|v_{k}\right\|_{V_{g}}^{2} \\
\quad \leq c\|f(s)\|_{V_{g}^{*}\left(\mathcal{O}_{\infty}\right)}^{2}+c\left(\left|z\left(\theta_{s} \omega\right)\right|^{4}+\left|z\left(\theta_{s} \omega\right)\right|^{2}\right),
\end{gathered}
$$

where $C_{g}:=\frac{v}{2}\left(1-\frac{4 v\|\nabla g\|_{\infty}}{m_{0} \lambda_{0}^{1 / 2}}\right)>0$ in view of Assumption G. 
Multiplying (53) by $e^{\int_{s}^{\tau}-2 \lambda+c_{0} \varepsilon\left|z\left(\theta_{r} \omega\right)\right| d r}$, then integrating the result w.r.t. $s \in(\tau-t, \sigma)$ and replacing $\omega$ by $\theta_{-\tau} \omega$, we find that

$$
\begin{aligned}
& \left\|v_{k}\left(\sigma, \tau-t, \theta_{-\tau} \omega, v_{\tau-t, k}\right)\right\|_{g}^{2}+C_{g} \int_{\tau-t}^{\sigma} e^{\int_{s}^{\sigma}-2 \lambda+c_{0} \varepsilon\left|z\left(\theta_{r-\tau} \omega\right)\right| d r}\left\|v_{k}(s)\right\|_{V_{g}}^{2} d s \\
& \leq e^{2 \lambda(\tau-\sigma)} e^{-2 \lambda t+c_{0} \varepsilon \int_{-t}^{\sigma-\tau}\left|z\left(\theta_{r} \omega\right)\right| d r}\left\|v_{\tau-t, k}\right\|_{H_{g}\left(\mathcal{O}_{k}\right)}^{2} \\
& \quad+c e^{2 \lambda(\tau-\sigma)} \int_{-\infty}^{\sigma-\tau} e^{2 \lambda s+c_{0} \varepsilon \int_{s}^{0}\left|z\left(\theta_{r} \omega\right)\right| d r}\|f(s+\tau)\|_{V_{g}^{*}\left(\mathcal{O}_{\infty}\right)}^{2} d s \\
& \quad+c e^{2 \lambda(\tau-\sigma)} \int_{-\infty}^{\sigma-\tau} e^{2 \lambda s+c_{0} \varepsilon \int_{s}^{0}\left|z\left(\theta_{r} \omega\right)\right| d r}\left(\left|z\left(\theta_{s} \omega\right)\right|^{2}+\left|z\left(\theta_{s} \omega\right)\right|^{4}\right) d s \\
& \leq e^{2 \lambda(\tau-\sigma)}\left(e^{-2 \lambda t+c_{0} \varepsilon \int_{-t}^{\sigma-\tau}\left|z\left(\theta_{r} \omega\right)\right| d r}\left\|v_{\tau-t, k}\right\|_{H_{g}\left(\mathcal{O}_{k}\right)}^{2}+\rho(\sigma, \tau, \omega)\right) .
\end{aligned}
$$

Using (43), (23), and $\mathbb{P}$-a.s. $\rho(\sigma, \tau, \omega)>0$, there is $T>0$ such that, for all $t \geq T$ and $\sigma \in$ $[\tau-t, \tau]$

$$
\begin{aligned}
& e^{-2 \lambda t+c_{0} \varepsilon \int_{-t}^{\sigma-\tau}\left|z\left(\theta_{r} \omega\right)\right| d r}\left\|v_{\tau-t, k}\right\|_{H_{g}\left(\mathcal{O}_{k}\right)}^{2} \\
& \quad \leq e^{-2 \lambda t+c_{0} \varepsilon \int_{-t}^{0}\left|z\left(\theta_{r} \omega\right)\right| d r}\left\|D\left(\tau-t, \theta_{-t} \omega\right)\right\|_{H_{g}\left(\mathcal{O}_{\infty}\right)}^{2} \\
& \leq e^{C_{0}(\omega)} e^{-\frac{3}{2} \lambda t}\left\|D\left(\tau-t, \theta_{-t} \omega\right)\right\|_{H_{g}\left(\mathcal{O}_{\infty}\right)}^{2} \leq \rho(\sigma, \tau, \omega) .
\end{aligned}
$$

We substitute (55) into (54) to obtain (45) as desired. In addition, by (43),

$$
\begin{aligned}
\rho(\sigma, \tau, \omega) & \leq c \int_{-\infty}^{0} e^{2 \lambda s+c_{0} \varepsilon \int_{s}^{0}\left|z\left(\theta_{r} \omega\right)\right| d r}\left(\|f(s+\tau)\|_{V_{g}^{*}\left(\mathcal{O}_{\infty}\right)}^{2}+\left|z\left(\theta_{s} \omega\right)\right|^{2}+\left|z\left(\theta_{s} \omega\right)\right|^{4}\right) d s \\
& \leq c e^{C_{0}(\omega)} \int_{-\infty}^{0} e^{\frac{3}{2} \lambda s}\left(\|f(s+\tau)\|_{V_{g}^{*}\left(\mathcal{O}_{\infty}\right)}^{2}+\left|z\left(\theta_{s} \omega\right)\right|^{2}+\left|z\left(\theta_{s} \omega\right)\right|^{4}\right) d s,
\end{aligned}
$$

then both (16) and Assumption F imply $\rho(\sigma, \tau, \omega)<+\infty$.

\subsection{Weak equi-continuity}

In this subsection, we show the weak equi-continuity of the solution sequence $\left\{v_{k}\right\}$. This is different from the weak continuity for a single system as given by Rosa [19].

Lemma 4.3 Suppose $f \in L_{\text {loc }}^{2}\left(\mathbb{R}, V_{g}^{*}\left(\mathcal{O}_{\infty}\right)\right)$. Let $\tau \in \mathbb{R}, \omega \in \Omega$, and $v_{\tau, k} \in H_{g}\left(\mathcal{O}_{k}\right)$ such that the expansion $\widetilde{v_{\tau, k}} \rightarrow v_{\tau, \infty}$ weakly in $H_{g}\left(\mathcal{O}_{\infty}\right)$ as $k \rightarrow \infty$. Then the sequence $\tilde{v_{k}}$ of expanding solutions satisfies

$$
\begin{aligned}
& \tilde{v_{k}}\left(t, \tau, \omega, v_{\tau, k}\right) \rightarrow v_{\infty}\left(t, \tau, \omega, v_{\tau, \infty}\right) \quad \text { weakly in } H_{g}\left(\mathcal{O}_{\infty}\right), \\
& \widetilde{v_{k}}\left(\cdot, \tau, \omega, v_{\tau, k}\right) \rightarrow v_{\infty}\left(\cdot, \tau, \omega, v_{\tau, \infty}\right) \quad \text { weakly in } L^{2}\left(\tau, \tau+T ; V_{g}\left(\mathcal{O}_{\infty}\right)\right), \forall T>0 .
\end{aligned}
$$

Proof From Lemmas 4.2 and 2.3, we can prove that, for all $T>0$,

$\left\{\widetilde{v_{k}}\right\}_{k}$ is bounded in $L^{\infty}\left(\tau, \tau+T ; H_{g}\left(\mathcal{O}_{\infty}\right)\right) \cap L^{2}\left(\tau, \tau+T ; V_{g}\left(\mathcal{O}_{\infty}\right)\right)$. 
We rewrite (18) as follows:

$$
\begin{aligned}
\frac{d v_{k}}{d t}= & -v A_{g, k} v_{k}-\varepsilon v z\left(\theta_{t} \omega\right) A_{g, k} h_{k}-B_{g, k}\left(v_{k}+\varepsilon h_{k} z\left(\theta_{t} \omega\right)\right) \\
& -v R_{g, k} v_{k}-\varepsilon v z\left(\theta_{t} \omega\right) R_{g, k} h_{k}+\varepsilon z\left(\theta_{t} \omega\right) h_{k}+\left.P_{g, k} f(t)\right|_{\mathcal{O}_{k}},
\end{aligned}
$$

where $h_{k}=\left.P_{g, k} h\right|_{\mathcal{O}_{k}}$. Since the norms of the operators $A_{g, k}, B_{g, k}$ are bounded in $k$ and $R_{g, k}$ satisfies (9), respectively, it follows from Lemma 2.3 and Assumption $\mathrm{H}$ that

$$
\left\|\frac{d v_{k}}{d t}\right\|_{V_{g}^{*}\left(\mathcal{O}_{k}\right)} \leq c\left(1+\left|z\left(\theta_{t} \omega\right)\right|^{2}+\left|z\left(\theta_{t} \omega\right)\right|^{4}\right)\left(\left\|{\widetilde{v_{k}}}_{V_{g}\left(\mathcal{O}_{\infty}\right)}^{2}+\right\| f(t) \|_{V_{g}^{*}\left(\mathcal{O}_{\infty}\right)}^{2}\right)
$$

This together with $\left\|{\widetilde{v_{k}}}^{\prime}\right\|_{V_{g}^{*}\left(\mathcal{O}_{\infty}\right)}=\left\|v_{k}^{\prime}\right\|_{V_{g}^{*}\left(\mathcal{O}_{k}\right)}$ and (58) implies that

$$
\left\{\widetilde{v_{k}^{\prime}}\right\}_{k} \text { is bounded in } L^{2}\left(\tau, \tau+T, V_{g}^{*}\left(\mathcal{O}_{\infty}\right)\right) \text {. }
$$

For each $i \in \mathbb{N}$, according to Aubin's compactness theorem [2] and the compactness of $V_{g}\left(\mathcal{O}_{i}\right) \hookrightarrow \mathbb{L}^{2}\left(\mathcal{O}_{i}\right)$, we obtain that

$\left\{\left.\widetilde{v_{k}}\right|_{\mathcal{O}_{i}}\right\}_{k}$ is relatively compact in $L^{2}\left(\tau, \tau+T ; \mathbb{L}^{2}\left(\mathcal{O}_{i}\right)\right), \quad \forall T>0$.

From (58) and (59), by a diagonal process, we can extract an index subsequence $k^{*}$ of $k$ such that

$$
\begin{aligned}
& \widetilde{v_{k^{*}}} \rightarrow v \quad \text { weak-star in } L^{\infty}\left(\tau, \tau+T ; H_{g}\left(\mathcal{O}_{\infty}\right)\right) \\
& \text { weakly in } L^{2}\left(\tau, \tau+T ; V_{g}\left(\mathcal{O}_{\infty}\right)\right) \\
& \text { strongly in } L^{2}\left(\tau, \tau+T ; \mathbb{L}^{2}\left(\mathcal{O}_{i}\right)\right), \quad \forall T>0, \forall i \in \mathbb{N},
\end{aligned}
$$

for some

$$
v \in L^{\infty}\left(\tau, \tau+T ; H_{g}\left(\mathcal{O}_{\infty}\right)\right) \cap L^{2}\left(\tau, \tau+T ; V_{g}\left(\mathcal{O}_{\infty}\right)\right), \quad \forall T>0 .
$$

We now show that $v$ is a weak solution of Eq. (18) at $k=\infty$. Indeed, by the weak formulation (20), the expansion $\widetilde{v_{k^{*}}}$ satisfies that, for each $w \in V_{g}\left(\mathcal{O}_{\infty}\right)$ and $t>\tau$,

$$
\begin{aligned}
\left(\widetilde{v_{k^{*}}}(t), w\right)_{g}= & \left(\widetilde{v_{k^{*}}}(\tau), w\right)_{g}+\int_{\tau}^{t}\left\langle\widetilde{\left.f(s)\right|_{\mathcal{O}_{k^{*}}}}, g w\right\rangle d s+\varepsilon \int_{\tau}^{t} z\left(\theta_{s} \omega\right)\left(h, g \widetilde{v_{k^{*}}}\right) d s \\
& -v \int_{\tau}^{t}\left\langle A_{g, \infty} \widetilde{v_{k^{*}}}, w\right\rangle_{g} d s-\varepsilon v \int_{\tau}^{t} z\left(\theta_{s} \omega\right)\left\langle A_{g, \infty} h, w\right\rangle_{g} d s \\
& -\int_{\tau}^{t}\left\langle B_{g, \infty}\left(\widetilde{v_{k^{*}}}+\varepsilon h z\left(\theta_{s} \omega\right)\right), g w\right\rangle d s-v \int_{\tau}^{t}\left\langle R_{g, \infty} \widetilde{v_{k^{*}}}, g w\right\rangle d s \\
& -\varepsilon v \int_{\tau}^{t} z\left(\theta_{s} \omega\right)\left\langle R_{g, \infty} h, g w\right\rangle d s,
\end{aligned}
$$


and we only need to consider the convergence of the forcing term $f$ as $k^{*} \rightarrow \infty$. Since $f \in L^{2}\left(\tau, t ; V_{g}^{*}\left(\mathcal{O}_{\infty}\right)\right)$ and $w \in V_{g}\left(\mathcal{O}_{\infty}\right)$, we have

$$
\begin{aligned}
\int_{\tau}^{t}\left\langle f(s)-\widetilde{\left.f(s)\right|_{\mathcal{O}^{*}}}, g w\right\rangle d s & =\int_{\tau}^{t} \int_{\mathcal{O}_{\infty} \backslash \mathcal{O}_{k^{*}}} f(s, x) \cdot w g d x d s \\
& \leq M_{0} \int_{\tau}^{t}\|f(s)\|_{V_{g}^{*}\left(\mathcal{O}_{\infty}\right)} d s\left(\int_{\mathcal{O}_{\infty} \backslash \mathcal{O}_{k^{*}}}|D w(x)|^{2} g d x\right)^{\frac{1}{2}} \\
& \leq c M_{0}\left(\int_{\tau}^{t}\|f(s)\|_{V_{g}^{*}\left(\mathcal{O}_{\infty}\right)}^{2} d s\right)^{\frac{1}{2}}\left(\int_{\mathcal{O}_{\infty} \backslash \mathcal{O}_{k^{*}}}|D w(x)|^{2} g d x\right)^{\frac{1}{2}} \\
& \leq c\left(\int_{\mathcal{O}_{\infty} \backslash \mathcal{O}_{k^{*}}}|D w(x)|^{2} g d x\right)^{\frac{1}{2}} \rightarrow 0 \text { as } k^{*} \rightarrow \infty .
\end{aligned}
$$

By taking the limit of (61) as $k^{*} \rightarrow \infty$ and noticing that $A_{g, \infty}, B_{g, \infty}, R_{g, \infty}$ are continuous operators, we see that $v$ is a weak solution of Eq. (18) with $k=\infty$. By the uniqueness of the solutions, we have $v(t)=v_{\infty}\left(t, \tau, \omega, v_{\tau, \infty}\right)$. Using a standard contradiction argument, we can show that the whole sequence $\left\{\widetilde{v}_{k}\right\}_{k}$ converges to $v_{\infty}$ in the sense of (60). This proves (57).

In addition, from the strong convergence in (60), we also have

$$
\left(\widetilde{v_{k}}(t), \xi\right)_{g} \rightarrow\left(v_{\infty}(t), \xi\right)_{g} \quad \text { for a.e. } t \in[\tau,+\infty) \text { and any } \xi \in \mathcal{V}\left(\mathcal{O}_{\infty}\right)
$$

On the other hand, for all $\xi \in \mathcal{V}\left(\mathcal{O}_{\infty}\right), \tau \leq t \leq t+a \leq \tau+T$ with $T>0$,

$$
\begin{aligned}
\left|\left(\widetilde{v_{k}}(t+a)-\widetilde{v_{k}}(t), \xi\right)_{g}\right| & =\int_{t}^{t+a}\left\langle\widetilde{v_{k}}(s), \xi\right\rangle_{g} d s=\int_{t}^{t+a} \int_{\mathcal{O}_{\infty}} \widetilde{v_{k}}(s) \xi g d x d s \\
& \leq\left(\int_{t}^{t+a} 1 d s\right)^{\frac{1}{2}}\left(\int_{t}^{t+a}\left(\int_{\mathcal{O}_{\infty}} \widetilde{v_{k}}(s) \xi g d x\right)^{2} d s\right)^{\frac{1}{2}} \\
& \leq a^{\frac{1}{2}} M_{0}^{\frac{1}{2}}\left(\int_{\mathcal{O}_{\infty}} \xi^{2} g d x\right)^{\frac{1}{2}}\left(\int_{t}^{t+a} \int_{\mathcal{O}_{\infty}}{\widetilde{v_{k}}}^{\prime 2}(s) d x d s\right)^{\frac{1}{2}} \\
& =a^{\frac{1}{2}} M_{0}^{\frac{1}{2}}\|\xi\|_{V_{g}\left(\mathcal{O}_{\infty}\right)}\left\|\widetilde{v_{k}}\right\|_{L^{2}\left(\tau, \tau+T ; V_{g}^{*}\left(\mathcal{O}_{\infty}\right)\right)} \\
& \leq C_{T} a^{\frac{1}{2}}\|\xi\|_{V_{g}\left(\mathcal{O}_{\infty}\right)},
\end{aligned}
$$

where $C_{T}$ is positive and independent of $k$. Thus, it follows from (58) and (63), we see that $\left\{\left(\widetilde{v_{k}}(t), \chi\right)\right\}$ is equi-bounded and equi-continuous on $[\tau, \tau+T]$ for all $T>0$. This together with (62) yields

$$
\left(\widetilde{v_{k}}(t), \xi\right) \rightarrow\left(v_{\infty}(t), \xi\right), \quad \forall t \in[\tau,+\infty), \forall \xi \in \mathcal{V}\left(\mathcal{O}_{\infty}\right)
$$

According to the density of $\mathcal{V}\left(\mathcal{O}_{\infty}\right)$ in $H_{g}\left(\mathcal{O}_{\infty}\right)$, we show (56).

By the similar (more simple) method as given in Lemma 4.3, we have weak continuity of each $v_{k}$. 
Corollary 1 For each fixed $k \in \overline{\mathbb{N}}$, if $v_{\tau, n} \rightarrow v_{\tau}$ weakly in $H_{g}\left(\mathcal{O}_{k}\right)$, then, as $n \rightarrow \infty$,

$$
\begin{array}{ll}
v_{k}\left(t, \tau, \omega, v_{\tau, n}\right) \rightarrow v_{k}\left(t, \tau, \omega, v_{\tau}\right) & \text { weakly in } H_{g}\left(\mathcal{O}_{k}\right), \\
v_{k}\left(\cdot, \tau, \omega, v_{\tau, n}\right) \rightarrow v_{k}\left(\cdot, \tau, \omega, v_{\tau}\right) & \text { weakly in } L^{2}\left(\tau, \tau+T ; V_{g}\left(\mathcal{O}_{k}\right)\right) \forall T>0 .
\end{array}
$$

\section{Equi-asymptotic compactness}

In this section, we establish an energy equation (as (75) below) for the expanding solution $\widetilde{v_{k}}$ and then we use this energy equation to verify the equi-asymptotic compactness of the sequence $\left\{\widetilde{\Phi_{k}}\right\}_{k}$ of expanding cocycles.

Theorem 5.1 Let Assumptions $\mathrm{F}, \mathrm{H}$, G hold true. Then, for any initial data $\left.v_{0, k} \in D\right|_{\mathcal{O}_{k}}(\tau-$ $\left.t_{k}, \theta_{-t_{k}} \omega\right)$ with $t_{k} \rightarrow+\infty, \tau \in \mathbb{R}, \omega \in \Omega$, and $D \in \mathfrak{D}$, the sequence of expanded solutions

$$
\left\{\widetilde{\Phi_{k}}\left(t_{k}, \tau-t_{k}, \theta_{-t_{k}} \omega\right) \widetilde{v_{0, k}}\right\}=\left\{\widetilde{v_{k}}\left(\tau, \tau-t_{k}, \theta_{-\tau} \omega, v_{0, k}\right)\right\}
$$

for problem (18) has a convergent subsequence in $H_{g}\left(\mathcal{O}_{\infty}\right)$.

Proof By taking $\sigma=\tau$ in (45), there is $k_{0} \in \mathbb{N}$ such that

$$
\sup _{k \geq k_{0}}\left\|v_{k}\left(\tau, \tau-t_{k}, \theta_{-\tau} \omega, v_{0, k}\right)\right\|_{H_{g}\left(\mathcal{O}_{k}\right)}^{2} \leq \rho(\tau, \tau, \omega)<+\infty,
$$

and thus, by Lemma 2.3(1), the sequence of expanding solutions

$$
\left\{\widetilde{v_{k}}\left(\tau, \tau-t_{k}, \theta_{-\tau} \omega, v_{0, k}\right)\right\}_{k} \text { is bounded in } H_{g}\left(\mathcal{O}_{\infty}\right)
$$

Passing to subsequence, there is $v \in H_{g}\left(\mathcal{O}_{\infty}\right)$ such that

$$
\widetilde{v_{k}}\left(\tau, \tau-t_{k}, \theta_{-\tau} \omega, v_{0, k}\right) \rightarrow v \quad \text { weakly in } H_{g}\left(\mathcal{O}_{\infty}\right)
$$

By the resonance theorem,

$$
\liminf _{k \rightarrow \infty}\left\|\widetilde{v_{k}}\left(\tau, \tau-t_{k}, \theta_{-\tau} \omega, v_{0, k}\right)\right\|_{H_{g}\left(\mathcal{O}_{\infty}\right)} \geq\|v\|_{H_{g}\left(\mathcal{O}_{\infty}\right)}
$$

Hence, in order to prove the weak convergence in (67) is strongly convergent, it suffices to prove that, for a subsequence,

$$
\limsup _{k \rightarrow \infty}\left\|\tilde{v_{k}}\left(\tau, \tau-t_{k}, \theta_{-\tau} \omega, v_{0, k}\right)\right\|_{H_{g}\left(\mathcal{O}_{\infty}\right)} \leq\|v\|_{H_{g}\left(\mathcal{O}_{\infty}\right)}
$$

By the cocycle property, we have, for each $m, k \in \mathbb{N}$,

$$
\widetilde{v_{k}}\left(\tau, \tau-t_{k}, \theta_{-\tau} \omega, v_{0, k}\right)=\widetilde{v_{k}}\left(\tau, \tau-m, \theta_{-\tau} \omega, \widetilde{v_{k}}\left(\tau-m, \tau-t_{k}, \theta_{-\tau} \omega, v_{0, k}\right)\right) .
$$

For each $m \in \mathbb{N}$, by taking $\sigma=\tau-m$ in (45), there is $K_{m} \in \mathbb{N}$ such that

$$
\sup _{k \geq K_{m}}\left\|\widetilde{v_{k}}\left(\tau-m, \tau-t_{k}, \theta_{-\tau} \omega, v_{0, k}\right)\right\|_{H_{g}\left(\mathcal{O}_{\infty}\right)}^{2} \leq e^{2 \lambda m} \rho(\tau-m, \tau, \omega)<+\infty,
$$


which means that the sequence $\left\{\widetilde{v_{k}}\left(\tau-m, \tau-t_{k}, \theta_{-\tau} \omega, v_{0, k}\right)\right\}_{k}$ is bounded in $H_{g}\left(\mathcal{O}_{\infty}\right)$ and thus there are $v^{m} \in H_{g}\left(\mathcal{O}_{\infty}\right)$ and an index subsequence $\{k(m)\}$ of $\{k(m-1)\}$ such that

$$
\widetilde{v_{k(m)}}\left(\tau-m, \tau-t_{k(m)}, \theta_{-\tau} \omega, v_{0, k(m)}\right) \rightarrow v^{m} \quad \text { weakly in } H_{g}\left(\mathcal{O}_{\infty}\right)
$$

We still use $\{k\}$ to denote the index diagonal subsequence $\{k(k)\}$. Then, as $k \rightarrow \infty$,

$$
\widetilde{v_{k}}\left(\tau-m, \tau-t_{k}, \theta_{-\tau} \omega, v_{0, k}\right) \rightarrow v^{m} \quad \text { weakly in } H_{g}\left(\mathcal{O}_{\infty}\right) \text { for all } m \in \mathbb{N} \text {. }
$$

By (70), (71), and (56), we have

$$
\widetilde{v_{k}}\left(\tau, \tau-t_{k}, \theta_{-\tau} \omega, v_{0, k}\right) \rightarrow v_{\infty}\left(\tau, \tau-m, \theta_{-\tau} \omega, v^{m}\right) \quad \text { in } H_{g}\left(\mathcal{O}_{\infty}\right)
$$

From (67), (72), and the uniqueness of weak limit, we have

$$
\nu_{\infty}\left(\tau, \tau-m, \theta_{-\tau} \omega, v^{m}\right)=v
$$

Now, we infer from (46) an energy equation $\mathcal{O}_{k}$ for all $k \in \overline{\mathbb{N}}$ :

$$
\begin{aligned}
\frac{d}{d t} & \left\|v_{k}\right\|_{g}^{2}+2 \lambda\left\|v_{k}\right\|_{g}^{2}+\Psi\left(v_{k}\right)+2 \varepsilon v z\left(\theta_{t} \omega\right)\left\langle A_{g, k} h_{k}, g v_{k}\right\rangle \\
& +2 \varepsilon v z\left(\theta_{t} \omega\right)\left\langle R_{g, k} h_{k}, g v_{k}\right\rangle+b_{g, k}\left(v_{k}+\varepsilon h_{k} z\left(\theta_{t} \omega\right), v_{k}+\varepsilon h_{k} z\left(\theta_{t} \omega\right), v_{k}\right) \\
= & 2\left\langle\left. f(t)\right|_{\mathcal{O}_{k}}, g v_{k}\right\rangle+2 \varepsilon z\left(\theta_{t} \omega\right)\left(h_{k}, g v_{k}\right)
\end{aligned}
$$

where $\Psi\left(v_{k}\right)=2 v\left\|v_{k}\right\|_{V_{g}\left(\mathcal{O}_{k}\right)}^{2}+2 v\left\langle R_{g, k} v_{k}, g v_{k}\right\rangle-2 \lambda\left\|v_{k}\right\|_{g}^{2}$.

Since the null-expansion does not change the norm, we have

$$
\Psi\left(v_{k}\right)=\Psi\left(\widetilde{v_{k}}\right), \quad \text { and } \quad\left\langle\left. f(t)\right|_{\mathcal{O}_{k}}, g v_{k}\right\rangle=\left\langle f(t), g \widetilde{v_{k}}\right\rangle .
$$

Hence, we can rewrite (74) on $\mathcal{O}_{\infty}$ as follows:

$$
\begin{gathered}
\frac{d}{d t}\left\|\widetilde{v_{k}}\right\|_{g}^{2}+2 \lambda\left\|\widetilde{v_{k}}\right\|_{g}^{2}+\Psi\left(\widetilde{v_{k}}\right)+2 \varepsilon v z\left(\theta_{t} \omega\right)\left\langle\widetilde{A_{g, k} h_{k}}, g \widetilde{v_{k}}\right\rangle+2 \varepsilon v z\left(\theta_{t} \omega\right)\left\langle\widetilde{R_{g, k} h_{k}}, g \widetilde{v_{k}}\right\rangle \\
+b_{g, \infty}\left(\widetilde{v_{k}}+\varepsilon h z\left(\theta_{t} \omega\right), \widetilde{v_{k}}+\varepsilon h z\left(\theta_{t} \omega\right), \widetilde{v_{k}}\right)=2\left\langle f(t), g \widetilde{v_{k}}\right\rangle+2 \varepsilon z\left(\theta_{t} \omega\right)\left(h, g \widetilde{v_{k}}\right)
\end{gathered}
$$

for all $k \in \overline{\mathbb{N}}$, where we regard $\widetilde{v_{\infty}}$ as $v_{\infty}$. Multiplying (75) by $e^{2 \lambda t}$ and then integrating the result over $[s, \tau]$, we obtain

$$
\begin{aligned}
\left\|\widetilde{v_{k}}\left(\tau, s, \omega, v_{s, k}\right)\right\|_{g}^{2}= & e^{2 \lambda(s-\tau)}\left\|\widetilde{s_{s, k}}\right\|_{g}^{2}-\int_{s}^{\tau} e^{\lambda(r-\tau)} \Psi\left(\widetilde{v_{k}}\left(r, s, \omega, v_{s, k}\right)\right) d r \\
& -2 v \varepsilon \int_{s}^{\tau} e^{2 \lambda(r-\tau)} z\left(\theta_{r} \omega\right)\left(\widetilde{A_{g, k} h_{k}}, g \widetilde{v_{k}}\left(r, s, \omega, v_{s, k}\right)\right\rangle d r \\
& -2 v \varepsilon \int_{s}^{\tau} e^{2 \lambda(r-\tau)} z\left(\theta_{r} \omega\right)\left(\widetilde{R_{g, k} h_{k}}, g \widetilde{v_{k}}\left(r, s, \omega, v_{s, k}\right)\right\rangle d r \\
& -2 \int_{s}^{\tau} e^{2 \lambda(r-\tau)} b_{g, \infty}\left(\widetilde{v_{k}}\left(r, s, \omega, v_{s, k}\right)+\operatorname{chz}\left(\theta_{r} \omega\right), \widetilde{v_{k}}\left(r, s, \omega, v_{s, k}\right)\right.
\end{aligned}
$$




$$
\begin{aligned}
& \left.+\varepsilon h z\left(\theta_{r} \omega\right), \widetilde{v_{k}}\left(r, s, \omega, v_{s, k}\right)\right) d r \\
& +2 \int_{s}^{\tau} e^{2 \lambda(r-\tau)}\left(f(r), g \widetilde{v_{k}}\left(r, s, \omega, v_{s, k}\right)\right\rangle d r \\
& +2 \varepsilon \int_{s}^{\tau} e^{2 \lambda(r-\tau)} z\left(\theta_{r} \omega\right)\left(h, g \widetilde{v_{k}}\left(r, s, \omega, v_{s, k}\right)\right) d r .
\end{aligned}
$$

Let $s=\tau-m$ and $\widetilde{v_{s, k}}=\widetilde{v_{k}}\left(\tau-m, \tau-t_{k}, \theta_{-\tau} \omega, v_{0, k}\right)$ in (76), we infer from (70) that, for all $k \in \mathbb{N}$,

$$
\begin{aligned}
& \left\|\widetilde{v_{k}}\left(\tau, \tau-t_{k}, \theta_{-\tau} \omega, v_{0, k}\right)\right\|_{g}^{2} \\
& =\left\|\widetilde{v_{k}}\left(\tau, \tau-m, \theta_{-\tau} \omega, \widetilde{v_{k}}\left(\tau-m, \tau-t_{k}, \theta_{-\tau} \omega, v_{0, k}\right)\right)\right\|_{g}^{2} \\
& =e^{-2 \lambda m}\left\|\widetilde{v_{k}}\left(\tau-m, \tau-t_{k}, \theta_{-\tau} \omega, v_{0, k}\right)\right\|_{g}^{2} \\
& -\int_{\tau-m}^{\tau} e^{2 \lambda(r-\tau)} \Psi\left(\widetilde{v_{k}}\left(r, \tau-m, \theta_{-\tau} \omega, \widetilde{v_{k}}\left(\tau-m, \tau-t_{k}, \theta_{-\tau} \omega, v_{0, k}\right)\right)\right) d r \\
& -2 v \varepsilon \int_{\tau-m}^{\tau} e^{2 \lambda(r-\tau)} \\
& \times z\left(\theta_{r-\tau} \omega\right)\left\langle\widehat{A_{g, k} h_{k}}, g \widetilde{v_{k}}\left(r, \tau-m, \theta_{-\tau} \omega, \widetilde{v_{k}}\left(\tau-m, \tau-t_{k}, \theta_{-\tau} \omega, v_{0, k}\right)\right)\right\rangle d r \\
& -2 \nu \varepsilon \int_{\tau-m}^{\tau} e^{2 \lambda(r-\tau)} \\
& \times z\left(\theta_{r-\tau} \omega\right)\left\langle\widehat{R_{g, k} h_{k}}, g \widetilde{v_{k}}\left(r, \tau-m, \theta_{-\tau} \omega, \widetilde{v_{k}}\left(\tau-m, \tau-t_{k}, \theta_{-\tau} \omega, v_{0, k}\right)\right)\right\rangle d r \\
& -2 \int_{\tau-m}^{\tau} e^{2 \lambda(r-\tau)} b_{g, k}\left(\tilde{u_{k}}(r, \tau-m), \tilde{u_{k}}(r, \tau-m), \tilde{u_{k}}(r, \tau-m)-\varepsilon h z\left(\theta_{r-\tau} \omega\right)\right) d r \\
& +2 \int_{\tau-m}^{\tau} e^{2 \lambda(r-\tau)}\left\langle f(r), g \widetilde{v_{k}}\left(r, \tau-m, \theta_{-\tau} \omega, \widetilde{v_{k}}\left(\tau-m, \tau-t_{k}, \theta_{-\tau} \omega, v_{0, k}\right)\right)\right\rangle d r \\
& +2 \varepsilon \int_{\tau-m}^{\tau} e^{2 \lambda(r-\tau)} \\
& \times z\left(\theta_{r-\tau} \omega\right)\left(h, g \widetilde{v_{k}}\left(r, \tau-m, \theta_{-\tau} \omega, \widetilde{v_{k}}\left(\tau-m, \tau-t_{k}, \theta_{-\tau} \omega, v_{0, k}\right)\right)\right) d r
\end{aligned}
$$

We then consider the limits of the right-hand side of (77) as $k \rightarrow \infty$ one by one. For the first term, by (45) with $\sigma=\tau-m$ and $t=t_{k}$, we see that

$$
\begin{aligned}
& e^{-2 \lambda m}\left\|\widetilde{v_{k}}\left(\tau-m, \tau-t_{k}, \theta_{-\tau} \omega, v_{0, k}\right)\right\|_{H_{g}\left(\mathcal{O}_{\infty}\right)}^{2} \\
& \leq e^{-\frac{3}{2} \lambda t_{k}}\left\|\widetilde{v_{0, k}}\right\|_{H_{g}\left(\mathcal{O}_{\infty}\right)}^{2}+c \int_{-\infty}^{-m} e^{2 \lambda s+c_{0} \varepsilon \int_{s}^{0}\left|z\left(\theta_{r} \omega\right)\right| d r}\|f(s+\tau)\|_{V_{g}^{*}(\mathcal{O})}^{2} d s \\
& \quad+c \int_{-\infty}^{-m} e^{2 \lambda s+c_{0} \varepsilon \int_{s}^{0}\left|z\left(\theta_{r} \omega\right)\right| d r}\left(\left|z\left(\theta_{s} \omega\right)\right|^{2}+\left|z\left(\theta_{s} \omega\right)\right|^{4}\right) d s
\end{aligned}
$$

Since $\left.v_{0, k} \in D\right|_{\mathcal{O}_{k}}\left(\tau-t_{k}, \theta_{-t_{k}} \omega\right)$, we have

$$
\begin{aligned}
e^{-\frac{3}{2} \lambda t_{k}}\left\|\widetilde{v_{0, k}}\right\|_{H_{g}\left(\mathcal{O}_{\infty}\right)}^{2} & =e^{-\frac{3}{2} \lambda t_{k}}\left\|v_{0, k}\right\|_{H_{g}\left(\mathcal{O}_{k}\right)}^{2} \leq e^{-\frac{3}{2} \lambda t_{k}}\left\|D_{\mid \mathcal{O}_{k}}\left(\tau-t_{k}, \theta_{-t_{k}} \omega\right)\right\|_{H_{g}\left(\mathcal{O}_{k}\right)}^{2} \\
& \leq e^{-\frac{3}{2} \lambda t_{k}}\left\|D\left(\tau-t_{k}, \theta_{-t_{k}} \omega\right)\right\|_{H_{g}\left(\mathcal{O}_{\infty}\right)}^{2} \rightarrow 0 \quad \text { as } k \rightarrow \infty
\end{aligned}
$$


Hence, we have the following estimate of the limit:

$$
\begin{aligned}
\limsup _{k \rightarrow \infty} e^{-2 \lambda m}\left\|\widetilde{v_{k}}\left(\tau-m, \tau-t_{k}, \theta_{-\tau} \omega, v_{0, k}\right)\right\|^{2} \\
\leq c \int_{-\infty}^{-m} e^{2 \lambda s+c_{0} \varepsilon \int_{s}^{0}\left|z\left(\theta_{r} \omega\right)\right| d r}\|f(s+\tau)\|_{V_{g}^{*}(\mathcal{O})}^{2} d s \\
\quad+c \int_{-\infty}^{-m} e^{2 \lambda s+c_{0} \varepsilon \int_{s}^{0}\left|z\left(\theta_{r} \omega\right)\right| d r}\left(\left|z\left(\theta_{s} \omega\right)\right|^{2}+\left|z\left(\theta_{s} \omega\right)\right|^{4}\right) d s .
\end{aligned}
$$

For the second term, we claim that $\Psi\left(v_{k}\right)=2 v\left\|v_{k}\right\|_{V_{g}\left(\mathcal{O}_{k}\right)}^{2}+2 \nu\left\langle R_{g, k} v_{k}, g v_{k}\right\rangle-2 \lambda\left\|v_{k}\right\|_{g}^{2}$ defines a norm which is equivalent to the norm in $V_{g}\left(\mathcal{O}_{k}\right)$. Indeed, by Lemma 2.1 and Assumption $\mathrm{G}$, we see that

$$
\begin{aligned}
\Psi\left(v_{k}\right) & \leq 2 v\left\|v_{k}\right\|_{V_{g}\left(\mathcal{O}_{k}\right)}^{2}+\frac{2 v\|\nabla g\|_{\infty}}{m_{0} \lambda_{0}^{1 / 2}}\left\|v_{k}\right\|_{V_{g}\left(\mathcal{O}_{k}\right)}^{2} \leq 2 v\left\|v_{k}\right\|_{V_{g}\left(\mathcal{O}_{k}\right)}^{2}+\frac{v}{2}\left\|v_{k}\right\|_{V_{g}\left(\mathcal{O}_{k}\right)}^{2} \\
& =\frac{5 v}{2}\left\|v_{k}\right\|_{V_{g}\left(\mathcal{O}_{k}\right)}^{2} .
\end{aligned}
$$

On the other hand, by the uniform Poincaré inequality and $\lambda=\frac{1}{3} \lambda_{0} \nu$,

$$
\Psi\left(v_{k}\right) \geq 2 v\|v\|_{V_{g}\left(\mathcal{O}_{k}\right)}^{2}-\frac{v}{2}\left\|v_{k}\right\|_{V_{g}\left(\mathcal{O}_{k}\right)}^{2}-2 \lambda \frac{1}{\lambda_{0}}\left\|v_{k}\right\|_{V_{g}\left(\mathcal{O}_{k}\right)}^{2}=\frac{5 v}{6}\left\|v_{k}\right\|_{V_{g}\left(\mathcal{O}_{k}\right)}^{2} .
$$

Thus, by the Fatou lemma and weak equi-continuity (57), we obtain

$$
\begin{aligned}
& \liminf _{k \rightarrow \infty} \int_{\tau-m}^{\tau} e^{2 \lambda(r-\tau)} \Psi\left(\widetilde{v_{k}}\left(r, \tau-m, \theta_{-\tau} \omega, \widetilde{v_{k}}\left(\tau-m, \tau-t_{k}, \theta_{-\tau} \omega, v_{0, k}\right)\right)\right) d r \\
& \quad \geq \int_{\tau-m}^{\tau} e^{2 \lambda(r-\tau)} \liminf _{k \rightarrow \infty} \Psi\left(\widetilde{v_{k}}\left(r, \tau-m, \theta_{-\tau} \omega, \widetilde{v_{k}}\left(\tau-m, \tau-t_{k}, \theta_{-\tau} \omega, v_{0, k}\right)\right)\right) d r \\
& \quad \geq \int_{\tau-m}^{\tau} e^{2 \lambda(r-\tau)} \Psi\left(v_{\infty}\left(r, \tau-m, \theta_{-\tau} \omega, v^{m}\right)\right) d r
\end{aligned}
$$

which implies

$$
\begin{aligned}
& \limsup _{k \rightarrow \infty}-\int_{\tau-m}^{\tau} e^{2 \lambda(r-\tau)} \Psi\left(\widetilde{v_{k}}\left(r, \tau-m, \theta_{-\tau} \omega, \widetilde{v_{k}}\left(\tau-m, \tau-t_{k}, \theta_{-\tau} \omega, v_{0, k}\right)\right)\right) d r \\
& \quad \leq-\int_{\tau-m}^{\tau} e^{2 \lambda(r-\tau)} \Psi\left(v_{\infty}\left(r, \tau-m, \theta_{-\tau} \omega, v^{m}\right)\right) d r .
\end{aligned}
$$

Similarly, from Lemma 4.3 and [5], we get

$$
\begin{gathered}
\limsup _{k \rightarrow \infty}-2 \int_{\tau-m}^{\tau} e^{2 \lambda(r-\tau)} b_{g, k}\left(\tilde{u_{k}}(r, \tau-m), \tilde{u_{k}}(r, \tau-m), \tilde{u_{k}}(r, \tau-m)-\varepsilon h z\left(\theta_{r-\tau} \omega\right)\right) d r \\
\quad \leq-2 \int_{\tau-m}^{\tau} e^{2 \lambda(r-\tau)} b_{g, \infty}\left(u_{\infty}(r, \tau-m), u_{\infty}(r, \tau-m), v_{\infty}\left(r, \tau-m, \theta_{-\tau} \omega, v^{m}\right)\right) d r .
\end{gathered}
$$


From (50) and the weak equi-continuity (57), we have

$$
\begin{aligned}
\underset{k \rightarrow \infty}{\limsup }- & \int_{\tau-m}^{\tau} e^{2 \lambda(r-\tau)} \\
& \left.\times z\left(\theta_{r-\tau} \omega\right) \widehat{\left(A_{g, k} h_{k}\right.}, g \widetilde{v_{k}}\left(r, \tau-m, \theta_{-\tau} \omega, \widetilde{v_{k}}\left(\tau-m, \tau-t_{k}, \theta_{-\tau} \omega, v_{0, k}\right)\right)\right\rangle d r \\
\leq & -\int_{\tau-m}^{\tau} e^{2 \lambda(r-\tau)} z\left(\theta_{r-\tau} \omega\right)\left\langle A_{g, \infty} h, g v_{\infty}\left(r, \tau-m, \theta_{-\tau} \omega, v^{m}\right)\right\rangle d r .
\end{aligned}
$$

By (51) and the weak equi-continuity (57), we get

$$
\begin{aligned}
\limsup _{k \rightarrow \infty} & -\int_{\tau-m}^{\tau} e^{2 \lambda(r-\tau)} \\
& \left.\times z\left(\theta_{r-\tau} \omega\right) \widehat{\left\langle R_{g, k} h_{k}\right.}, g \widetilde{v_{k}}\left(r, \tau-m, \theta_{-\tau} \omega, \widetilde{v_{k}}\left(\tau-m, \tau-t_{k}, \theta_{-\tau} \omega, v_{0, k}\right)\right)\right\rangle d r \\
\leq & -\int_{\tau-m}^{\tau} e^{2 \lambda(r-\tau)} z\left(\theta_{r-\tau} \omega\right)\left\langle R_{g, \infty} h, g v_{\infty}\left(r, \tau-m, \theta_{-\tau} \omega, v^{m}\right)\right\rangle d r .
\end{aligned}
$$

By the weak equi-continuity (57) again, it follows from (71), on $L^{2}\left(\tau-m, \tau ; V_{g}\left(\mathcal{O}_{\infty}\right)\right)$, that

$$
\widetilde{v_{k}}\left(\cdot, \tau-m, \theta_{-\tau} \omega, \widetilde{v_{k}}\left(\tau-m, \tau-t_{k}, \theta_{-\tau} \omega, v_{0, k}\right)\right) \rightarrow v_{\infty}\left(\cdot, \tau-m, \theta_{-\tau} \omega, v^{m}\right) .
$$

By $f \in L^{2}\left(\tau-m, \tau ; V_{g}^{*}\left(\mathcal{O}_{\infty}\right)\right)$ and Assumption $\mathrm{H}$, we know

$$
r \mapsto e^{2 \lambda(r-\tau)}\left(f(r)+\varepsilon z\left(\theta_{r-\tau} \omega\right) h\right) g \in L^{2}\left(\tau-m, \tau ; V_{g}^{*}\left(\mathcal{O}_{\infty}\right)\right)
$$

Thereby, we have

$$
\begin{aligned}
\lim _{k \rightarrow \infty} & 2 \int_{\tau-m}^{\tau} e^{2 \lambda(r-\tau)}\left\{f(r)+\varepsilon z\left(\theta_{r-\tau} \omega\right) h,\right. \\
& \left.g \widetilde{v_{k}}\left(r, \tau-m, \theta_{-\tau} \omega, \widetilde{v_{k}}\left(\tau-m, \tau-t_{k}, \theta_{-\tau} \omega, v_{0, k}\right)\right)\right\rangle d r \\
= & 2 \int_{\tau-m}^{\tau} e^{2 \lambda(r-\tau)}\left\{f(r)+\varepsilon z\left(\theta_{r-\tau} \omega\right) h, g v_{\infty}\left(r, \tau-m, \theta_{-\tau} \omega, v^{m}\right)\right\rangle d r .
\end{aligned}
$$

Taking the sup-limit of (77) as $k \rightarrow \infty$, from (78) to (83), we obtain

$$
\begin{aligned}
& \limsup _{k \rightarrow \infty}\left\|\widetilde{v_{k}}\left(\tau, \tau-t_{k}, \theta_{-\tau} \omega, v_{0, k}\right)\right\|^{2} \\
& \leq c \int_{-\infty}^{-m} e^{2 \lambda s+c_{0} \varepsilon \int_{s}^{0}\left|z\left(\theta_{r} \omega\right)\right| d r}\|f(s+\tau)\|_{V_{g}^{*}(\mathcal{O})}^{2} d s \\
& +c \int_{-\infty}^{-m} e^{2 \lambda s+c_{0} \varepsilon \int_{s}^{0}\left|z\left(\theta_{r} \omega\right)\right| d r}\left(\left|z\left(\theta_{s} \omega\right)\right|^{2}+\left|z\left(\theta_{s} \omega\right)\right|^{4}\right) d s \\
& -\int_{\tau-m}^{\tau} e^{2 \lambda(r-\tau)} \Psi\left(v_{\infty}\left(r, \tau-m, \theta_{-\tau} \omega, v^{m}\right)\right) d r \\
& -2 \nu \varepsilon \int_{\tau-m}^{\tau} e^{2 \lambda(r-\tau)} z\left(\theta_{r-\tau} \omega\right)\left\langle A_{g, \infty} h, g \nu_{\infty}\left(r, \tau-m, \theta_{-\tau} \omega, v^{m}\right)\right\rangle d r \\
& -2 \varepsilon v \int_{\tau-m}^{\tau} e^{2 \lambda(r-\tau)} z\left(\theta_{r-\tau} \omega\right)\left\langle R_{g, \infty} h, g v_{\infty}\left(r, \tau-m, \theta_{-\tau} \omega, v^{m}\right)\right\rangle d r
\end{aligned}
$$




$$
\begin{aligned}
& -2 \int_{\tau-m}^{\tau} e^{2 \lambda(r-\tau)} b_{g, \infty}\left(u_{\infty}(r, \tau-m), u_{\infty}(r, \tau-m), v_{\infty}\left(r, \tau-m, \theta_{-\tau} \omega, v^{m}\right)\right) d r \\
& +2 \int_{\tau-m}^{\tau} e^{2 \lambda(r-\tau)}\left\{f(r)+\varepsilon z\left(\theta_{r-\tau} \omega\right) h, g v_{\infty}\left(r, \tau-m, \theta_{-\tau} \omega, v^{m}\right)\right\rangle d r .
\end{aligned}
$$

We denote the sum of last five terms in (84) by $I(m)$, let $k=\infty, s=\tau-m$ in (76), and then we obtain

$$
\|v\|_{H_{g}\left(\mathcal{O}_{\infty}\right)}^{2}=\left\|v_{\infty}\left(\tau, \tau-m, \theta_{-\tau} \omega, v^{m}\right)\right\|_{H_{g}\left(\mathcal{O}_{\infty}\right)}^{2}=e^{-2 \lambda m}\left\|v^{m}\right\|_{g}^{2}+I(m)
$$

Substituting (85) into (84) yields, for all $m \in \mathbb{N}$,

$$
\begin{aligned}
& \limsup _{k \rightarrow \infty}\left\|\widetilde{v_{k}}\left(\tau, \tau-t_{k}, \theta_{-\tau} \omega, v_{0, k}\right)\right\|_{H_{g}\left(\mathcal{O}_{\infty}\right)}^{2} \\
& \leq\|v\|_{H_{g}\left(\mathcal{O}_{\infty}\right)}^{2}-e^{-2 \lambda m}\left\|v^{m}\right\|_{g}^{2}+c \int_{-\infty}^{-m} e^{2 \lambda s+c_{0} \varepsilon \int_{s}^{0}\left|z\left(\theta_{r} \omega\right)\right| d r}\|f(s+\tau)\|_{V_{g}^{*}(\mathcal{O})}^{2} d s \\
& \quad+c \int_{-\infty}^{-m} e^{2 \lambda s+c_{0} \varepsilon \int_{s}^{0}\left|z\left(\theta_{r} \omega\right)\right| d r}\left(\left|z\left(\theta_{s} \omega\right)\right|^{2}+\left|z\left(\theta_{s} \omega\right)\right|^{4}\right) d s .
\end{aligned}
$$

By Lemma 4.1 and Assumption F, as $m \rightarrow \infty$,

$$
\begin{aligned}
& \int_{-\infty}^{-m} e^{2 \lambda s+c_{0} \varepsilon \int_{s}^{0}\left|z\left(\theta_{r} \omega\right)\right| d r}\|f(s+\tau)\|_{V_{g}^{*}(\mathcal{O})}^{2} d s \\
& \leq e^{C_{0}(\omega)} \int_{-\infty}^{-m} e^{\frac{3}{2} \lambda s}\|f(s+\tau)\|_{V_{g}^{*}(\mathcal{O})}^{2} d s \rightarrow 0 .
\end{aligned}
$$

Therefore, letting $m \rightarrow \infty$ in (86), we obtain (69) as desired.

Corollary 2 Let $k \in \overline{\mathbb{N}}$ be fixed. Then, for any $\left.v_{0, n} \in D\right|_{\mathcal{O}_{k}}\left(\tau-t_{n}, \theta_{-t_{n}} \omega\right)$ with $t_{n} \rightarrow+\infty$, $D \in \mathfrak{D}, \tau \in \mathbb{R}$, and $\omega \in \Omega$, the sequence $\left\{v_{k}\left(\tau, \tau-t_{n}, \theta_{-\tau} \omega\right) v_{0, n}\right\}_{n}$ of solutions of (18) has a convergent subsequence in $H_{g}\left(\mathcal{O}_{k}\right)$.

\section{Final conclusion}

In the last section, we deduce the existence and large-domain stability of the attractor when the domain changes from bounded to unbounded.

Theorem 6.1 For each $k \in \overline{\mathbb{N}}$, let $\Phi_{k}$ be the cocycle associated with the $g$-NS equation (18) on $\mathcal{O}_{k}$, and let $\mathfrak{D}_{k}:=\left.\mathfrak{D}\right|_{\mathcal{O}_{k}}$ be the restriction of the universe $\mathfrak{D}$ in (22). Then $\Phi_{k}$ has a $\mathfrak{D}_{k}$ pullback random attractor $\mathcal{A}_{k}$ in $H_{g}\left(\mathcal{O}_{k}\right)$.

Proof By taking $\sigma=\tau$ in (45), we find that $\Phi_{k}$ has an absorbing set $\mathcal{M}_{k}$ given by

$$
\mathcal{M}_{k}(\tau, \omega)=\left\{u \in H_{g}\left(\mathcal{O}_{k}\right):\|u\|_{H_{g}\left(\mathcal{O}_{k}\right)} \leq \rho(\tau, \omega)\right\}, \quad \forall k \in \overline{\mathbb{N}}
$$

where $\rho(\tau, \omega):=\rho(\tau, \tau, \omega)=c\left(\rho_{1}(\tau, \omega)+\rho_{2}(\tau, \omega)\right)$ with

$$
\rho_{1}(\tau, \omega):=\int_{-\infty}^{0} e^{2 \lambda s+c_{0} \varepsilon \int_{s}^{0}\left|z\left(\theta_{r} \omega\right)\right| d r}\|f(s+\tau)\|_{V_{g}^{*}\left(\mathcal{O}_{\infty}\right)}^{2} d s
$$




$$
\rho_{2}(\tau, \omega):=\int_{-\infty}^{0} e^{2 \lambda s+c_{0} \varepsilon \int_{s}^{0}\left|z\left(\theta_{r} \omega\right)\right| d r}\left(\left|z\left(\theta_{s} \omega\right)\right|^{2}+\left|z\left(\theta_{s} \omega\right)\right|^{4}\right) d s
$$

We need to prove that $\rho_{1}$ and $\rho_{2}$ are tempered with the growth rate $\frac{3}{2} \lambda$. Indeed, by Lemma 4.1 and Assumption F,

$$
\begin{aligned}
& e^{-\frac{3}{2} \lambda t} \rho_{1}\left(\tau-t, \theta_{-t} \omega\right) \\
& \quad=e^{-\frac{3}{2} \lambda t} \int_{-\infty}^{0} e^{2 \lambda s+c_{0} \varepsilon \int_{s}^{0}\left|z\left(\theta_{r-t} \omega\right)\right| d r}\|f(s+\tau-t)\|_{V_{g}^{*}(\mathcal{O})}^{2} d s \\
& \quad \leq e^{-\frac{3}{2} \lambda t} \int_{-\infty}^{-t} e^{\frac{3}{2} \lambda(s+t)+c_{0} \varepsilon \int_{s}^{-t} \mid z\left(\theta_{r}(\omega) \mid d r\right.}\|f(s+\tau)\|_{V_{g}^{*}(\mathcal{O})}^{2} d s \\
& \leq e^{C_{0}(\omega)} \int_{-\infty}^{-t} e^{\lambda s}\|f(s+\tau)\|_{V_{g}^{*}(\mathcal{O})}^{2} d s \rightarrow 0
\end{aligned}
$$

as $t \rightarrow+\infty$. Similarly, the tempered property of $\left|z\left(\theta_{s} \omega\right)\right|^{2}+\left|z\left(\theta_{s} \omega\right)\right|^{4}$ implies that $\rho_{2}$ is tempered. Therefore, $\mathcal{M}_{k} \in \mathfrak{D}_{k}$.

On the other hand, by Corollary $2, \Phi_{k}$ is $\mathfrak{D}_{k}$-pullback asymptotically compact. Therefore, it follows from the abstract result [21] that $\Phi_{k}$ has a unique $\mathfrak{D}_{k}$-pullback random attractor denoted by $\mathcal{A}_{k}=\left\{\mathcal{A}_{k}(\tau, \omega)\right\}$.

In addition, by Theorem 3.1, the expanded cocycle $\widetilde{\Phi_{k}}$ has a $\widetilde{\mathfrak{D}_{k}}$-pullback random attractor in $H_{k}\left(\mathcal{O}_{\infty}\right)$. This expanded attractor is just the null-expansion $\widetilde{\mathcal{A}_{k}}$ of $\mathcal{A}_{k}$.

Finally, we establish the large-domain stability (upper-semicontinuity) of random attractors as $k \rightarrow \infty$.

Theorem 6.2 The sequence $\left\{\mathcal{A}_{k}\right\}_{k}$ of random attractors associated with problem (18) satisfies

$$
\operatorname{dist}_{H_{g}\left(\mathcal{O}_{\infty}\right)}\left(\widetilde{\mathcal{A}_{k}}(\tau, \omega), \mathcal{A}_{\infty}(\tau, \omega)\right) \rightarrow 0
$$

as $k \rightarrow \infty$ for all $\tau \in \mathbb{R}$ and $\omega \in \Omega$, where $\widetilde{\mathcal{A}_{k}}$ is the null-expansion of $\mathcal{A}_{k}$.

Proof The proof is similar to that of [16, Theorem VI.2] and so is omitted here.

\section{Acknowledgements}

The authors thank the referees for valuable comments and suggestions which improved the presentation of this manuscript.

\section{Funding}

Fuzhi Li was supported by the Science and Technology Foundation of Jiangxi Education Department (No. GJJ190880). Dongmei Xu was supported by the Research Project of Shangrao Normal University (No. 201905, 202019). Lianbing She was supported by the Science and Technology Foundation of Guizhou Province (No. [2020]1Y007) and School level Foundation of Liupanshui Normal University (LPSSYKJTD201907).

\section{Abbreviations}

Not applicable.

Availability of data and materials

Not applicable. 
Authors' contributions

Each of the authors contributed to each part of this study equally. All authors read and approved the final vision of the manuscript.

\section{Author details}

${ }^{1}$ School of Mathematics and Computer Science, Shangrao Normal University, Shangrao 334001, P.R. China. ${ }^{2}$ School of Mathematics and Information Engineering, Liupanshui Normal University, Liupanshui 553004, P.R. China.

\section{Publisher's Note}

Springer Nature remains neutral with regard to jurisdictional claims in published maps and institutional affiliations.

Received: 22 March 2020 Accepted: 9 July 2020 Published online: 23 July 2020

\section{References}

1. Arnold, L.: Random Dynamical Systems. Springer, Berlin (1998)

2. Aubin, J.P.: Un théeorème de compacité. C. R. Acad. Sci. Paris 256, 5042-5044 (1963)

3. Bae, H.O., Roh, J.: Existence of solutions of the g-Navier-Stokes equations. Taiwan. J. Math. 8(1), 85-102 (2004)

4. Brzeźniak, Z., Caraballo, T., Langa, J.A., Li, Y., Łukaszewicz, G., Real, J.: Random attractors for stochastic 2D-Navier-Stokes equations in some unbounded domains. J. Differ. Equ. 255(11), 3897-3919 (2013)

5. Brzeźniak, Z., Li, Y.: Asymptotic compactness and absorbing sets for $2 \mathrm{D}$ stochastic Navier-Stokes equations on some unbounded domains. Trans. Am. Math. Soc. 358(12), 5587-5629 (2006)

6. Cui, $\mathrm{H}$ :: Convergences of asymptotically autonomous pullback attractors towards semigroup attractors. Discrete Contin. Dyn. Syst., Ser. B 24(8), 3525-3535 (2019)

7. Cui, H., Kloeden, P.: Tail convergences of pullback attractors for asymptotically converging multi-valued dynamical systems. Asymptot. Anal. 112(3-4), 165-184 (2019)

8. Flandoli, F., Schmalfuss, B.: Random attractors for the 3D stochastic Navier-Stokes equation with multiplicative white noise. Stoch. Stoch. Rep. 59(1), 21-45 (1996)

9. Iftimie, D., Raugel, G.: Some results on the Navier-Stokes equations in thin 3D domains. J. Differ. Equ. 169(2), 281-331 (2001)

10. Jiang, J., Hou, Y.: The global attractor of $g$-Navier-Stokes equations with linear dampness on $\mathbb{R}^{2}$. Appl. Math. Comput. 215(3), 1068-1076 (2009)

11. Jiang, J., Hou, Y.: Pullback attractor of 2D non-autonomous $g$-Navier-Stokes equations on some bounded domains. Appl. Math. Mech. Engl. Ed. 31(6), 697-708 (2010)

12. Kloeden, P., Simsen, J.: Asymptotically autonomous multivalued Cauchy problems with spatially variable exponents. J. Math. Anal. Appl. 445(1), 513-531 (2017)

13. Langa, J.A., Łukaszewicz, G., Real, J.: Finite fractal dimension of pullback attractors for non-autonomous 2D Navier-Stokes equations in some unbounded domains. Nonlinear Anal. 66(3), 735-749 (2007)

14. Li, D., Lu, K., Wang, B., Wang, X.: Limiting behavior of dynamics for stochastic reaction-diffusion equations with additive noise on thin domains. Discrete Contin. Dyn. Syst. 38(1), 187-208 (2018)

15. Li, D., Lu, K., Wang, B., Wang, X.: Limiting dynamics for non-autonomous stochastic retarded reaction-diffusion equations on thin domains. Discrete Contin. Dyn. Syst. 39(7), 3717-3747 (2019)

16. Li, F., Li, Y.: Asymptotic behavior of stochastic $g$-Navier-Stokes equations on a sequence of expanding domains. J. Math. Phys. 60, 061505 (2019)

17. Raugel, G., Sell, G.R.: Navier-Stokes equations on thin 3D domains. I. Global attractors and global regularity of solutions. J. Am. Math. Soc. 6(3), 503-568 (1993)

18. Roh, J.: Dynamics of the g-Navier-Stokes equations. J. Differ. Equ. 211(2), 452-484 (2005)

19. Rosa, R.: The global attractor for the 2D Navier-Stokes flow on some unbounded domains. Nonlinear Anal. 32(1), 71-85 (1998)

20. Song, X., Sun, C., Yang, L.: Pullback attractors for 2D Navier-Stokes equations on time-varying domains. Nonlinear Anal. 45, 437-460 (2019)

21. Wang, B.: Sufficient and necessary criteria for existence of pullback attractors for non-compact random dynamical systems. J. Differ. Equ. 253(5), 1544-1583 (2012)

\section{Submit your manuscript to a SpringerOpen ${ }^{\circ}$ journal and benefit from:}

- Convenient online submission

- Rigorous peer review

Open access: articles freely available online

- High visibility within the field

- Retaining the copyright to your article

Submit your next manuscript at $\downarrow$ springeropen.com 